\title{
Equine skeletal muscle adaptations to exercise and training: evidence of differential regulation of autophagosomal and mitochondrial components
}

Kenneth Bryan ${ }^{1 \dagger}$, Beatrice A. McGivney ${ }^{1 \dagger}$, Gabriella Farries ${ }^{1}$, Paul A. McGettigan', Charlotte L. McGivney ${ }^{1}$, Katie F. Gough', David E. MacHugh ${ }^{1,2}$, Lisa M. Katz ${ }^{3}$ and Emmeline W. Hill ${ }^{1 *}$

\begin{abstract}
Background: A single bout of exercise induces changes in gene expression in skeletal muscle. Regular exercise results in an adaptive response involving changes in muscle architecture and biochemistry, and is an effective way to manage and prevent common human diseases such as obesity, cardiovascular disorders and type II diabetes. However, the biomolecular mechanisms underlying such responses still need to be fully elucidated. Here we performed a transcriptome-wide analysis of skeletal muscle tissue in a large cohort of untrained Thoroughbred horses $(n=51)$ before and after a bout of high-intensity exercise and again after an extended period of training. We hypothesized that regular high-intensity exercise training primes the transcriptome for the demands of high-intensity exercise.
\end{abstract}

Results: An extensive set of genes was observed to be significantly differentially regulated in response to a single bout of high-intensity exercise in the untrained cohort (3241 genes) and following multiple bouts of high-intensity exercise training over a six-month period (3405 genes). Approximately one-third of these genes (1025) and several biological processes related to energy metabolism were common to both the exercise and training responses. We then developed a novel network-based computational analysis pipeline to test the hypothesis that these transcriptional changes also influence the contextual molecular interactome and its dynamics in response to exercise and training. The contextual network analysis identified several important hub genes, including the autophagosomal-related gene GABARAPL1, and dynamic functional modules, including those enriched for mitochondrial respiratory chain complexes I and $V$, that were differentially regulated and had their putative interactions 're-wired' in the exercise and/or training responses.

Conclusion: Here we have generated for the first time, a comprehensive set of genes that are differentially expressed in Thoroughbred skeletal muscle in response to both exercise and training. These data indicate that consecutive bouts of high-intensity exercise result in a priming of the skeletal muscle transcriptome for the demands of the next exercise bout. Furthermore, this may also lead to an extensive 're-wiring' of the molecular interactome in both exercise and training and include key genes and functional modules related to autophagy and the mitochondrion.

Keywords: Equine, Skeletal muscle, Transcriptome, Exercise, Training, RNAseq, Functional module, Network analysis, Mitochondria, Autophagy

\footnotetext{
* Correspondence: emmeline.hill@ucd.ie

tEqual contributors

${ }^{1}$ UCD School of Agriculture and Food Science, University College Dublin,

Belfield D04 V1W8, Ireland

Full list of author information is available at the end of the article
} 


\section{Background}

Equine athletes have a genetic heritage that has been influenced by millions of years of evolution as grazing animals on prairie and steppe. More recently, centuries of intense selective breeding in the Thoroughbred horse has led to the refinement of multiple physiological adaptations for athletic performance, resulting in an ideal model of a natural athlete for the investigation of exercise and adaptive training responses. Equine skeletal muscle shows a remarkable ability to adapt to physical exercise and long-term training; however, the genetic, epigenetic and molecular changes underlying these adaptive responses have yet to be fully elucidated [1-3]. Cyclic muscle contraction during repeated bouts of exercise over time (training or conditioning) is known to induce physiological adaptation in skeletal muscle, which exhibits remarkable plasticity in structure and function [4]. In equine athletes, training generally leads to an increase in muscle mass and aerobic capacity but the specific response, in terms of muscle fibre type and metabolic adaptation, depends mainly on the type of training regime (e.g. endurance or sprint type exercise) [5-7], nutrition [8-10] and an individual's specific genetic potential [11-13]. Principal genetic determinants of muscle fibre type include the myostatin gene (MSTN), which encodes a ligand of the TGF-beta receptor family that negatively regulates muscle growth, and that has been associated with strength and performance in both human [14] and equine athletes [15]. Sequence polymorphisms in the equine MSTN gene are highly predictive of optimal race distance in Thoroughbred horses [13, 15-19]. Horses homozygous for the 'sprint' variant (C-allele or SINE insertion) have $12.5 \%$ more type $2 X$ myofibers than horses with the alternate allele [20]. Following a period of training in Thoroughbred horses an increase in type $2 A$ and a concurrent decrease in type $2 X$ fibres along with an overall increase in muscle mass is typically observed [6]. As type $2 A$ fibres can sustain high power outputs for longer than $2 X$ the functional implication of this is increased endurance. Concurrent with changes in muscle mass and fibre type, exercise training elicits metabolic adaptations. This primarily involves an increased capacity for oxidative phosphorylation [5], increased mitochondrial density [21] and a shift toward oxidizing proportionately more fats and less glucose during exercise [22].

It has been hypothesized that the adaptive response to training is caused by incremental changes in gene expression following a single bout of exercise, which will "accumulate" during the traing period leading to new baseline levels of gene expression. This would result in a significant overlap in the exercise and training response genes [23-25]. The alternate hypothesis is that transient differential expression of genes in response to exercise precedes adaptive changes through secondary mechanisms. In this case little overlap would be seen between the exercise and training response genes [26]. While there is an assumption that "accumulative" changes play a major role in the adaptive response no study has clearly demonstrated this. In human skeletal muscle the mRNA expression of key transcription factors is transiently induced by exercise training leading to increases in downstream transcriptional and mitochondrial proteins [25]. In equine athletes it has been shown that a single bout of high-intensity exercise consisting of an incremental step-test to fatigue elicits a modulation of the expression of genes involved in metabolism and muscle hypertrophy, signatures of endurance and resistance exercise, respectively [27]. Following a period of training the basal levels of genes related to the mitochondrion, oxidative phosphorylation and fatty acid metabolism have been shown to be significantly upregulated [28], supporting the hypothesis that training may cause a transcriptional reprogramming of the muscle.

A range of approaches has been taken to better understand the molecular adaptations to exercise and training with many factors needing to be considered for appropriate experimental design $[6,22,28-32]$. Generally, the analysis of the impact of an experimental variable (e.g. environment, treatment, mutation, disease etc.) on a cell, tissue or organism results in a list of statistically significant response variables, such as genes. It is common, owing to the modular architecture of biological systems, to then examine this list for statistical over-representation of known functional modules (e.g. pathways or complexes) to assist biological interpretation (often referred to pathway enrichment analysis, or similar). Although valuable, such analysis is confined by the limits of the current knowledge of functional modules which tends to be biased [33] and incomplete [34]. However, less supervised approaches that are informed more by the similarity of entity (i.e. gene) behaviour, are more open to uncovering unknown or context-specific gene relationships [34, 35].

The function of most genes, or gene products (proteins, miRNA, IncRNAs, etc.), can only be carried out in combination with other biomolecules as part of a functional module [36]. Therefore, to fully elucidate the functional relevance of a set of genes we must also model the related set of molecular interactions and their dynamics. There are several methods for direct detection $[37,38]$ or inferral [39-41] of molecular interactions. Once determined, the set of molecular interactions may be modelled as a network of nodes (genes) and edges (interactions) and interrogated with an extensive toolbox of established network analysis methods [34, 42]. Common metrics include the centrality indices 'degree' and 'betweeness', which are used to measure the importance of individual network nodes in terms of the local connections and their network wide influence respectively. In the context of biomolecular interaction networks, 
these 'hub' and 'bottleneck' nodes tend to be fundamentally important to the behaviour of the network [43] and indeed to the biological process, cell, tissue or organism as a whole [44]. Other higher level node grouping or clustering methods, such as 'community detection' [45, 46], allow modelling of the underlying functional modules (e.g. complex or pathway) in a particular molecular interaction network $[36,42]$. Other methods extend this concept further to analyse multi-state, or 'dynamic' networks recorded over various time points or experimental treatments [47].

Although hundreds of thousands of protein-protein interactions (PPIs) have been recorded across many organisms, cell types and experimental conditions and made publicly available [48], proteome-scale detection of PPIs still remains beyond the budget and expertise of most researchers. Genome-wide gene expression analysis, however, is now relatively affordable and commonplace. Thus, high-throughput gene expression data generated for an experimental context is often integrated with the universal set of known molecular interactions (i.e. the global interactome) to infer putative contextual molecular interaction networks. However, most implementations of this approach only attempt to predict the contextual state of the nodes in the network (e.g. the set of genes expressed in or associated with the condition of interest) and include all associated PPIs as edges, regardless of the experimental conditions or cell types in which they were detected, which is clearly a gross over-simplification [49-51]. It has been recognized for over a decade that genes that exhibit highly correlated co-expression profiles, as determined by Pearson's correlation coefficient for example, often interact within the same biological module $[40,52]$. This raises the prospect of using gene expression information to also infer the contextual state of the edges in a molecular interaction network but this in itself is hampered by exceedingly high false positive rates [41]. Here we have developed a novel pipeline to integrate contextual information, derived from gene expression data, with publicly available PPI data [48] to predict both the nodes and edges in the contextual PPI network. Ultimately, this more refined contextual model should lead to an improved elucidation of the interactions and functional modules that are active in each of the experimental conditions considered in this study.

In summary, the aim of this study was to investigate changes in the transcriptome of the skeletal muscle of untrained Thoroughbred horses (UR) in response to a single bout of high-intensity sprint exercise (UE), and following an exercise conditioning (training) regime (TR). We hypothesised that regular bouts of highintensity exercise training would prime the transcriptome of Thoroughbred skeletal muscle for the demands of the next exercise bout. Furthermore, by integrating this information on contextual transcriptional changes with known molecular interactions, we also tested the hypothesis that these transcriptional adaptions may lead to a 're-wiring' of the molecular interactome in response to high-intensity exercise and training. Lastly we have demonstrated that a refined computational networkbased approach, which considers both context-specific nodes (genes) and edges (interactions), has the potential to uncover novel features indicative of specific biological processes when compared to standard supervised approaches.

\section{Methods}

\section{Study cohort}

University College Dublin Animal Research Ethics Committee approval, a licence from the Department of Health (B100/3525) and explicit owner/trainer informed consent were obtained for the use of all horses and procedures in this study.

The study cohort comprised a subset of Thoroughbred horses, trained for Flat racing at a single training establishment under the management of a single trainer. The UR cohort comprised of yearling Thoroughbred horses ( $n=51 ; 23$ males and 28 females; $19.5 \pm 1.5$ months old) in submaximal training prior to entering sprint training. The UE cohort consisted of the same group of horses undertaking their first or second 'work day' (WD, highintensity sprint exercise simulating a race) on an allweather gallop. The TR cohort consisted of horses at the end of the racing season following approximately six months of sprint training. All horses in this group had achieved "race fitness" with an average of $15.1 \pm 9.1 \mathrm{SD}$ WDs (range 6-43). Table 1 summarises details of sampling and physiological measurements.

\section{Tissue sampling time-points}

All resting skeletal muscle samples (see protocol below) were collected between 7:30 am and 11:30 am. UE samples were collected from a subset of the UR horses ( $n=46 ; 23$ males and 23 females; $24.6 \pm 2.3$ months old) approximately four hours following high-intensity exercise. The study cohort comprised horses in active race training and therefore the number of biopsies that could be sampled was limited. A single $4 \mathrm{~h}$ post-exercise timepoint was selected that represented the time previously shown to have the greatest number of differentially expressed genes [27] and the greatest magnitude of effect on transcripts in equine skeletal muscle postexercise [30]. In addition, the time point was selected to avoid potential disruption to routine activity on the yard.

TR samples were collected at rest following a sprint training period of approximately six months from a subset of the UR horses $(n=22$; eight males, and 14 females; $31.1 \pm 1.5$ months old). Only horses that were 
Table 1 Cohort information and experimental conditions

\begin{tabular}{|c|c|c|c|}
\hline & $\begin{array}{l}\text { Untrained Rest } \\
\text { (UR) }\end{array}$ & $\begin{array}{l}\text { Untrained Exercise } \\
\text { (UE) }\end{array}$ & $\begin{array}{l}\text { Trained Rest } \\
\text { (TR) }\end{array}$ \\
\hline$n$ & 22 & 17 & 22 \\
\hline Male & 8 & 8 & 8 \\
\hline Female & 16 & 9 & 16 \\
\hline Mean age in days (Range) & $590(513-673)$ & $765(649-896)$ & $947.6(884-1051)$ \\
\hline \multicolumn{4}{|l|}{ Exercise parameters (WD1): } \\
\hline -Mean Peak Velocity $(\mathrm{m} / \mathrm{s})$ & . & $16.2(14-17.1)$ & . \\
\hline -Mean Peak Heart Rate (bpm) & & $224(212-233)$ & . \\
\hline -Mean Peak Plasma Lactate (mmol/l) & . & $26.7(20.8-32.2)$ & . \\
\hline Mean sub-maximal training weeks prior to sampling (canter) & 0 & $4.7(1.6-13.1)$ & $27.7(10.1-47.0)$ \\
\hline Mean work days prior to sampling: & 0 & 1 & $15.1(6-43)$ \\
\hline$n=0$ & 22 & & \\
\hline$n=1$ & 0 & 17 & 0 \\
\hline$n>1$ & 0 & 0 & 22 \\
\hline$>5 n \leq 11$ & 0 & 0 & 8 \\
\hline$>10 n \leq 20$ & 0 & 0 & 9 \\
\hline$n>20$ & 0 & 0 & 5 \\
\hline
\end{tabular}

Number of individuals ( $n$ ), males and females, and mean age are given for experimental cohorts Untrained Rest (UR), Untrained Exercise (UE) and Trained Rest (TR). Details of exercise and training regimens for each Thoroughbred cohort prior to sampling day are also shown. Sampling of the Untrained Exercise (UE) cohort was performed $\sim 4 \mathrm{~h}$ post-exercise on the first work day (WD1). Sampling of the Trained Rest (TR) cohort was performed after an average of $\sim 15 \mathrm{WDs}$ over a six-month training period

sampled as part of the UR cohort and had a matching sample in the UE or the TR cohort were used in subsequent analysis. The final experimental cohorts contained $n=22$ (UR), $n=17$ (UE) and $n=22$ (TR) horses. Summary statistics are shown in Table 1 .

\section{Exercise and training protocols}

Horses exercised six days per week, with gradual introduction and increased frequency of WDs, following which horses entered competitive racing. Training was modified based on soundness, fitness and aptitude, with all decisions made by a single trainer. Prior to exercise, each horse was fitted with a heart rate (HR) telemetry system (Polar Equine S810i heart rate monitor system) and global positioning system (GPS, GPSports Systems SPI10, Canberra, Australia) which recorded speed, HR and exercise distance. The WD exercise protocol was as follows: horses were warmed-up on a horse walker for $10 \mathrm{~min}$ (walk and trot) and then walked under saddle for 5-10 min. On the gallop, horses walked for $300 \mathrm{~m}$, trotted for $700 \mathrm{~m}$, walked for approximately $100 \mathrm{~m}$ and then galloped up to a maximum velocity for approximately 500-800 m (average distance of 698.8 $\pm 223.9 \mathrm{~m}$ ).

\section{Biopsy sampling and RNA sequencing protocols}

Percutaneous needle muscle biopsies (approx. $300 \mathrm{mg}$ ) were obtained from the ventral compartment of the middle gluteal muscle from standing unsedated horses using a previously described method [53] and preserved in RNAlater (Thermo Fisher, Massachusetts, United States). Total RNA was extracted from approximately $70 \mathrm{mg}$ tissue, using a protocol combining TRIzol reagent (Thermo Fisher, Massachusetts, United States), DNase treatment (RNase free DNase) (Qiagen, Hilden, Germany) and RNeasy Mini-Kit (Qiagen). RNA was quantified using a Nano Drop ND1000 spectrophotometer V 3.5.27 and RNA quality and purity were assessed using the $18 \mathrm{~S} / 28 \mathrm{~S}$ ratio and RNA integrity number (RIN) on an Agilent Bioanalyser with the RNA 6000 Nano LabChip kit6 (Agilent, Cork, Ireland). The RNA isolated from UR, UE and TR samples had an average RIN of 8.1 (7.2-9.3), 8.2 (7-9.1) and 8.1 (5.4-8.6), respectively. RNA sequencing was performed by the Research Technology Support Facility Michigan State University. Indexed, strandspecific Illumina sequencing libraries were prepared using the TruSeq Stranded mRNA Library Preparation Kit LT (Illumina, San Diego, United States). Libraries were pooled with ten indexed libraries per pool and sequenced on an Illumina HiSeq 2500 using Rapid Run flow cell and reagents (Illumina). Dual lane loading was employed, meaning a single pool was loaded across both lanes of the flow cell. Each pool was sequenced on one flow cell (two lanes). Sequencing was performed in a $2 \times 100 \mathrm{bp}$ paired end (PE100) format. Sequence data was demultiplexed and converted to FastQ format files. 


\section{RNA-seq data pre-processing}

FASTQC [54] was used to assess sequencing quality and STAR [55] was used to align reads to the horse reference genome (Ensembl release 62). SAMtools [56] was used to convert files from Sam to sorted indexed Bam format and featureCounts [57] was used to assign reads to exons. The correlation between lanes (two technical replicates) for each sample was checked to ensure a correlation of $>99 \%$ prior to merging data. Differentially expressed (DE) genes were identified using the limma [58] $\mathrm{R}$ package. Count data was scale normalised using the calcNormFactors function from the EdgeR [59] package. The voom transformation was applied to the normalised data and a linear model was fitted to the data using the horse as a blocking factor to take into account the pair effect. The empirical Bayes method was used to calculate $P$ values. Ensembl IDs of small RNAs (BioMart biotypes: rRNA, snRNA, misc_RNA, SnoRNA, miRNA) were first removed from the dataset. Differentially expressed (DE) genes were defined as those that were significantly upregulated or downregulated (Benjamini-Hochberg corrected $P$-value $<0.05$ ) and that were upregulated or downregulated by at least $25 \%$ between the two cohorts being compared (i.e. UE versus UR, TR versus UR).

\section{Statistical over-representation analysis}

To facilitate a complete downstream analysis pipeline, equine Ensembl IDs were first uniquely mapped (one-toone relationship only) to their better annotated human orthologs, retrieved from the BioMart database [60]. All subsequent analyses were performed with these human orthologs and their annotations. Over-representation of the gene ontology (GO) categories [61] and KEGG and Reactome pathway annotations [62, 63] was performed in an automated manner using the RDAVIDWebService [64]. The expected or background gene list used in the over-representation analysis included all genes that had a one-to-one mapping with human orthologs and were expressed in skeletal muscle during at least one time point $(n=14,688)$. The statistical significance of gene category over-representation was calculated based on the DAVID software EASE score ( $P$-value based on a modified Fisher Exact t-test) [65] and corrected for multiple testing using the Benjamini-Hochberg stepdown correction.

\section{Construction of putative contextual molecular interaction networks}

Two dynamic gene interaction networks were constructed, the first to model the molecular interactions before and after exercise in untrained horses (UR vs. UE) and the second to model the training response (UR vs. TR). In each case the set of nodes (genes) in the dynamic network were confined to those that had been found to be differentially expressed in the respective response. An edge (gene interaction) was drawn between nodes (genes) $a$ and $b$ if (i) there was any previous experimental evidence of a molecular interaction and (ii) if their expression levels were found to be significantly correlated in the respective cohort (i.e. UR, UE or TR). Experimentally validated protein-protein interactions (PPIs) for human, that were annotated to the IMEx (International Molecular Exchange consortium) standard [66], were then obtained (12-09-2016) from the IntAct database [48] using the PSICQUIC web-service [67] (see Additional file 1 for the specific query used). These interactions were recorded across many cell types, tissues and experimental conditions and represent the global set of all possible interactions in human as defined by IntAct DB. Gene expression correlations were calculated across all samples within the relevant cohort (i.e. UR, UE or TR) using Spearman's rank correlation and only positive, statistically significant correlations (i.e. greater than the minimum critical value given the number of samples in the cohort) were used to generate contextual edges.

\section{Network analysis}

Network analysis and visualization was implemented in the $\mathrm{R}$ statistical programming language and primarily used tools from the igraph package [68]. The most influential' nodes (genes) in each network were determined based on both degree (edge number) and betweeness (number of shortest paths that pass through that node) [69]. These metrics highlight the nodes (genes) that have the most influence on information flow in the network at a local (i.e. hubs) and global (i.e. bottlenecks) level respectively. Hubs are scored based simply on node degree (number of edges attached to a node) and bottlenecks are scored based on the node betweeness (number of shortest paths that pass through a node). The modular or systems level architecture of a network was interrogated in an unsupervised manner (i.e. based only on network topology) by partitioning the nodes (genes) into groups such that the edge (gene interaction) density was higher within groups than between. This was performed using the fastgreedy community detection method, which also utilizes edge weights (based on expression correlation in this case) [45] and was also implemented via igraph. In practice, we retained only non-trivial clusters (i.e. containing two or more genes) and validated these clusters (putative functional modules) by functional enrichment analysis based on pathways and GO terms (implemented as before).

\section{Results}

Identification of genes and known functional categories associated with the exercise and training responses

We applied both a fold-change and multiple test correction cut-off to identify differentially expressed genes in the 
exercise (UR vs. UE) and training (UR vs. TR) responses. We identified 3241 genes that were significantly (BH-corrected $P$-value $<0.05)$ differentially expressed $(>1.25$-fold $)$ and mapped uniquely to human orthologs in the exercise response (Additional file 2: Table S1). A similar number of genes (3405) were associated with the training response (Additional file 2: Table S2). Approximately one third of the exercise response genes (1025) were also associated with the training response and $95 \%$ of genes were expressed in the same direction (relative to UR) in both responses. There were 2216 and 2380 genes uniquely associated with the exercise or training response respectively.

To attempt to uncover the underlying biological processes involved in the adaptation of skeletal muscle to exercise and training, the gene lists derived from differentially expressed transcripts were assessed for statistical overrepresentation of known functional modules based on five functional annotation schemas. Two schemas related to biological pathways (curated by KEGG [63] and Reactome [62] databases) and three related to Cellular Component (CC), Molecular Function (MF) and Biological Process (BP) gene ontology (GO) sub-categories [61].

For the exercise response gene list several KEGG and Reactome pathways were found to be significantly overrepresented and were observed to fall into one of three general themes, namely: energy metabolism (Integration of energy metabolism: $P=4.97 \times 10^{-11}$, Diabetes pathways: $P=3.57 \times 10^{-06}$, Oxidative phosphorylation: $P=0.0047$ and Pyruvate Metabolism and TCA Cycle: $P=0.0204$ ); muscle contraction (Muscle contraction: $P=3.57 \times 10^{-06}$, Cardiac muscle contraction: $P=0.0222$ ) and hemostasis (Hemostasis: $P=3.76 \times 10$ ${ }^{-05}$, Signaling by PDGF: $\left.P=0.0167\right)$. There was also significant enrichment for several pathways related to neurodegenerative diseases (Alzheimer's Disease: $P=4.95 \times 10$ ${ }^{-06}$, Huntington's Disease: $9.15 \times 10^{-06}$ and Parkinson's Disease: $2.75 \times 10^{-05}$ ). Statistical enrichment was also observed for similar themes for the GO Biological Process sub-category (e.g. Cellular respiration: $P=0.0004$, Muscle contraction: $P=0.0004$, Vasculature development: $P=0.0307)$. GO term enrichment also provided more refined annotation relating the Cellular Component subcategory (e.g. Mitochondrion: $P=4.14 \times 10^{-15}$, Contractile fibre: $P=6.40 \times 10^{-11}$, Mitochondrial respiratory chain: $\left.P=7.41 \times 10^{-05}\right)$ and the Molecular Function subcategory (e.g. Cytoskeletal protein binding: $P=1.41 \times 10^{-06}$, Actin binding: $P=0.0006$ ).

The functional modules related to muscle contraction were the most downregulated modules associated with the exercise response gene list. For example, the largest overrepresented module, Contractile fibre, contained 56 genes, which were mostly $(44 / 56)$ downregulated (mean: -1.52fold) in exercise relative to the untrained rest cohort. These muscle contraction related modules were also found to be unique to the exercise response (i.e. this theme was not found to be associated with the training response, see below). The top twenty most significantly over-represented functional modules in the exercise response gene list are provided in Additional file 2: Table S3 and illustrated via five barcharts (one per annotation schema) in Fig. 1.

This analysis was also repeated for the exercise-specific subset of 2216 genes (i.e. exclusively associated with the exercise response relative to the training response gene list). KEGG pathways Dilated cardiomyopathy and Hypertrophic cardiomyopathy remained marginally statistically significantly over-represented $(P=0.0252$ and $P=0.0472$ resp. $)$. Reactome pathways Muscle contraction $\left(P=1.52 \times 10^{-05}\right)$ and Hemostasis $(P=0.0031)$ and Transmembrane transport of small molecules $(P=0.0034)$ also remained significantly over-represented. Significant enrichment for the Lysosome $(P=0.0437)$ pathway, which was not found in the full exercise response gene list, was also observed. Several gene ontology sub-category terms also remained statistically significant, such as Cytoskeletal binding $\left(P=4.32 \times 10^{-05}\right)$, Contractile fibre $\left(P=1.12 \times 10^{-06}\right)$ and Membrane lipid metabolic process $(P=0.0057)$. The top twenty most significantly over-represented functional modules for this exercise-specific gene list are given in Additional file 2: Table S4 and illustrated in Fig. 2.

For the training response gene list (3405 genes) there was significant enrichment for several KEGG and Reactome pathways related to energy metabolism (e.g. Oxidative phosphorylation: $P=3.59 \times 10^{-08}$, Citrate cycle $P=5.12 \times 10^{-05}$, Integration of energy metabolism: $P=4.66 \times 10^{-14}$, Diabetes pathways: $P=3.10 \times 10^{-08}$, Pyruvate metabolism: $P=2.93 \times 10^{-07}$ ). Additionally, several KEGG and Reactome pathways related to signal transduction (e.g. Signaling by GPCR: $P=8.94 \times 10^{-14}$, Olfactory transduction: $P=7.68 \times 10^{-12}$, Neuroactive ligand-receptor interaction: $P=0.0001$, Calcium signaling pathway: $P=0.0190$, Signaling by FGFR: $P=0.0169$ and Complement and coagulation cascades: $P=0.03$ ) were also significantly over-represented in the training response. There was also a significant enrichment for neurodegenerative disease associated KEGG pathways (e.g. Alzheimer's Disease: $P=6.57 \times 10^{-06}$, Huntington's Disease: $4.5 \times 10^{-05}$ and Parkinson's Disease: $2.56 \times 10^{-13}$ ). Gene ontology term analysis for the training response also revealed a highly significant over-representation of neurological processes (Neurological system process: $P=4.85 \times 10^{-27}$, Cognition: $P=1.92 \times 10^{-22}$, Sensory perception: $P=4.21 \times 10^{-21}$ ) and the extracellular region (Extracellular region: $P=1.40 \times 10^{-10}$ ). Mitochondrial related molecular functions and components were also significantly over-represented (Mitochondrial respiratory chain: $P=1.45 \times 10^{-08}$, Mitochondrial inner membrane: $P=2.53 \times 10^{-08}, N A D H$ dehydrogenasel Respiratory chain complex I: 


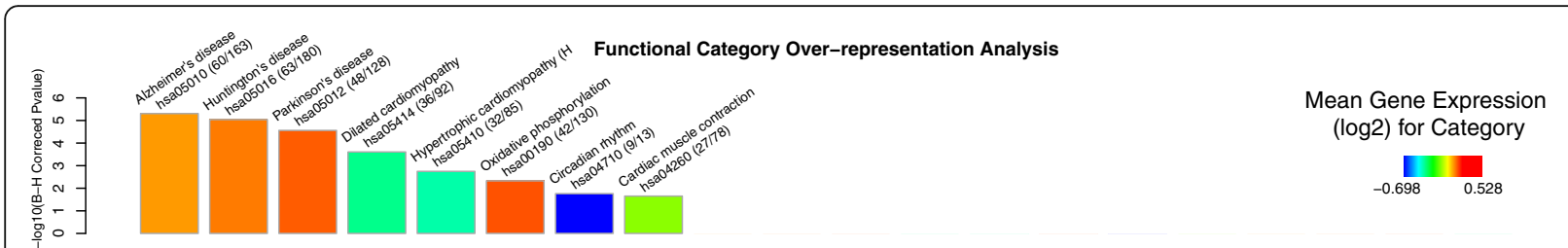

KEGG Pathways
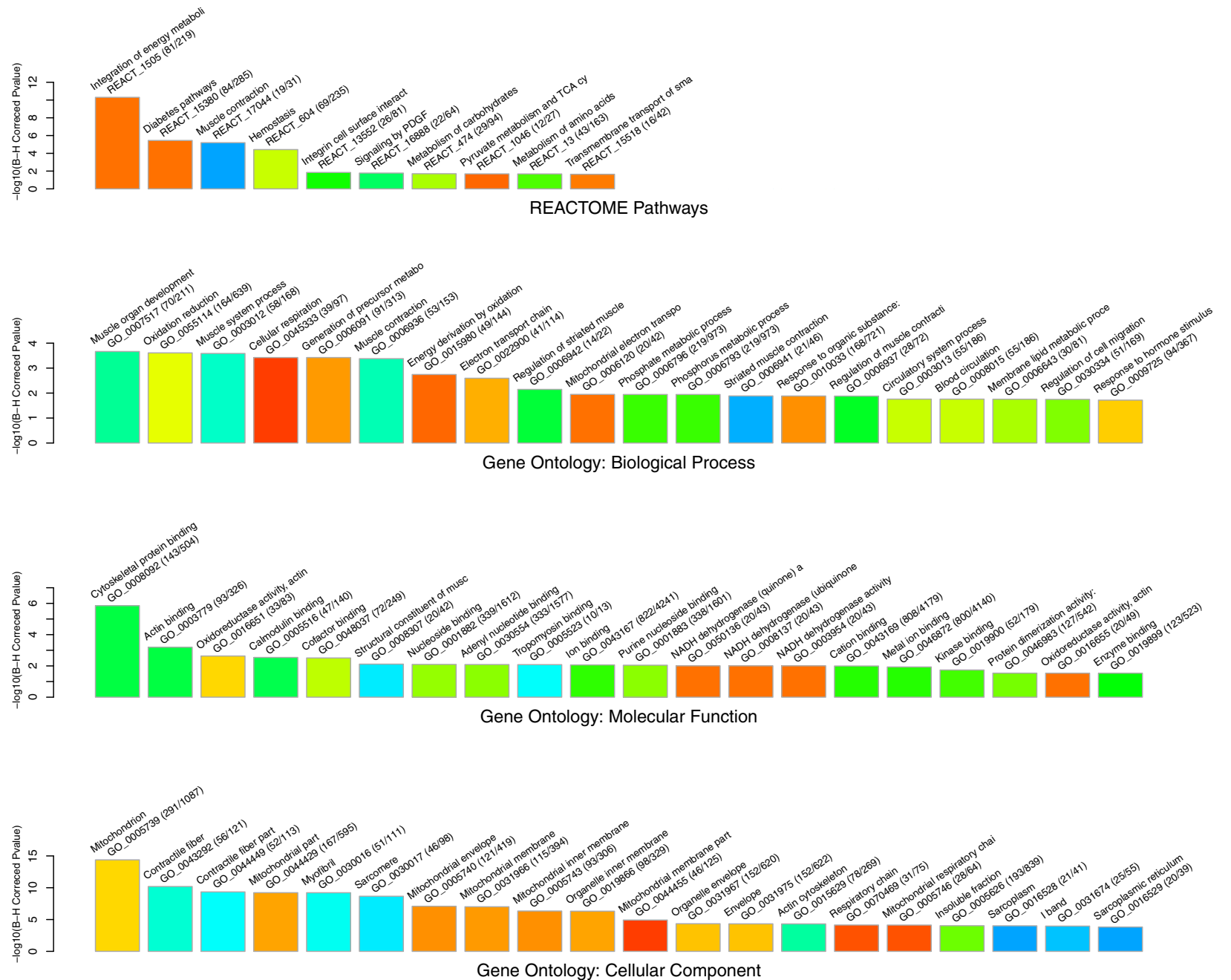

Fig. 1 Cellular functions of the exercise response. Bar charts showing over-representation of functional categories (described by KEGG and REACTOME pathways and Gene Ontology: Biological Process, Molecular Function and Cellular Component) for the list of genes $(n=3241)$ that showed statistically significant differential expression, of at least $+/-1.25$-fold, between the muscle tissue (g/uteus medius) from the untrained rest (UR) and the untrained exercise (UE) Thoroughbred cohorts. Bars represent the most significant functional modules (up to 20) for each of the five annotation schemas. Bar height represents statistical significance (- $\log _{10}$ transformed Benjamini-Hochberg (B-H) Corrected P-value) of the over-representation, based on the EASE-score (conservative Fisher Exact t-test). Bar color represents the mean differential expression ( $\log _{2}($ UE/UR) for the genes in this module (see color key). Category name, ID and size (number category genes in gene list/category size) are given above each bar. For example it can be seen that the fourth most significant Gene Ontology:Biological Process is 'Cellular respiration' and that it is one of the most up-regulated functional categories on average (red color) and that 39 out of the 97 genes assigned to this category are differentially expressed between UE and UR cohorts. Full results are provided in Additional file 2: Table S3

$P=1.29 \times 10^{-06}$, Mitochondrial proton-transporting ATP synthase complex: $P=0.0007)$ in the training response gene list. The top twenty most significantly over-represented functional modules for the training response are given in Additional file 2: Table S5 and illustrated in Fig. 3.

This analysis was repeated for the training-specific subset of 2380 genes that were uniquely associated with 


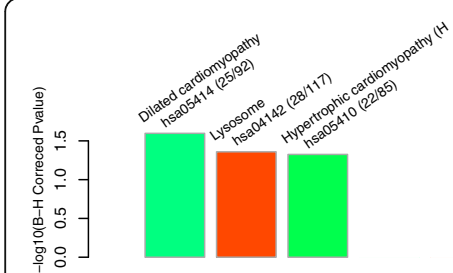

Functional Category Over-representation Analysis

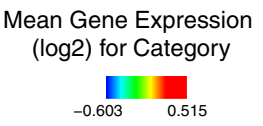

KEGG Pathways

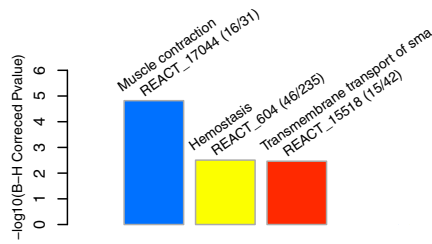

REACTOME Pathways
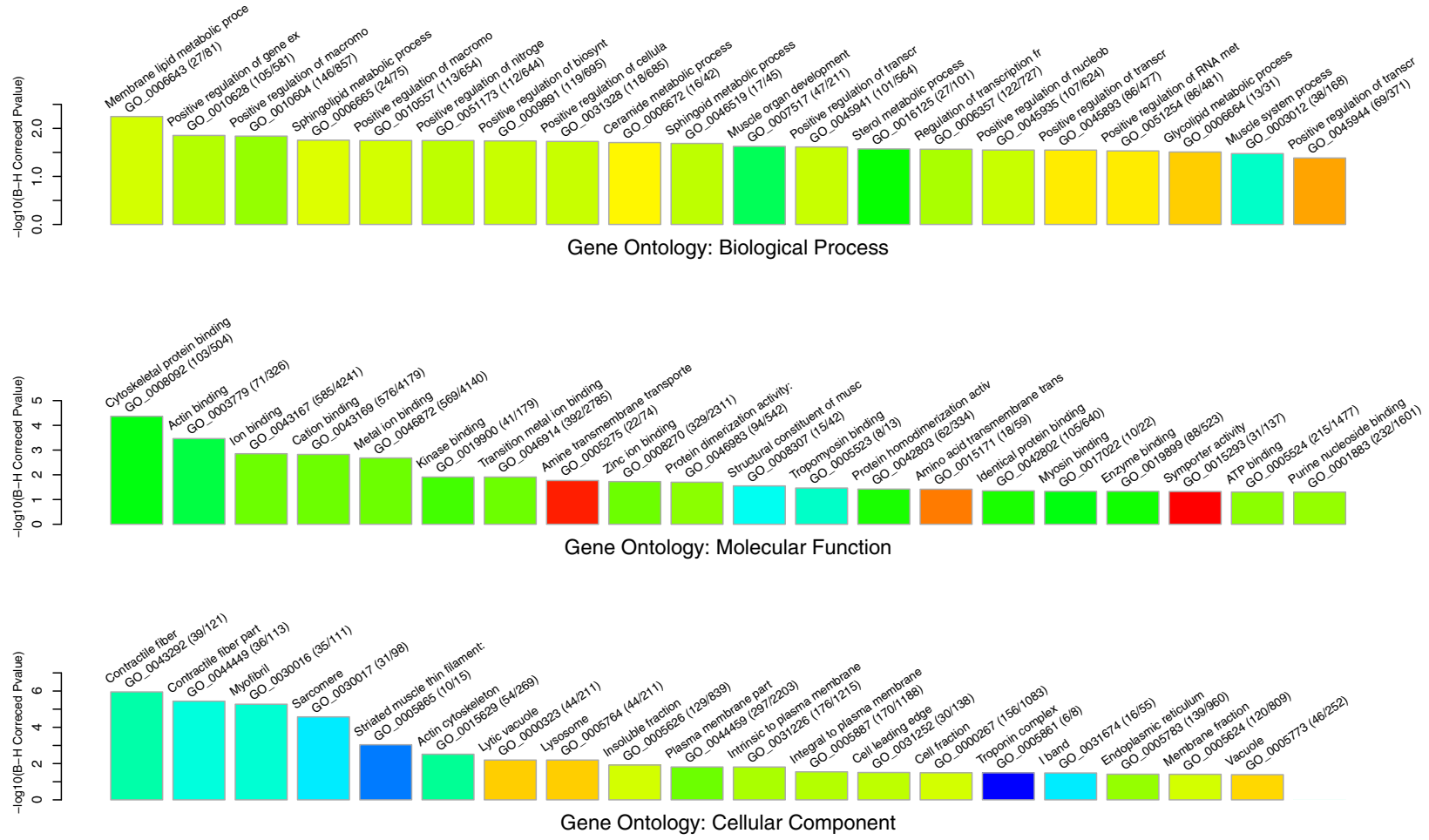

Fig. 2 Cellular functions of the exercise-specific response. Bar charts showing over-representation of functional categories (described by KEGG and REACTOME pathways and Gene Ontology: Biological Process, Molecular Function and Cellular Component) for the list of genes ( $n=2216)$ that showed statistically significant differential expression, of at least $+/-1.25$-fold, between the muscle tissue (gluteus medius) from the untrained rest (UR) and the untrained exercise (UE) but not the trained rest (TR) Thoroughbred cohorts. Bars represent the most significant functional modules (up to 20 ) for each of the five annotation schemas. Bar height represents statistical significance $\left(-\log _{10}\right.$ transformed Benjamini-Hochberg (B-H) Corrected $P$-value) of the over-representation, based on the EASE-score (conservative Fisher Exact t-test). Bar color represents the mean differential expression ( $\log _{2}(\mathrm{UE} / \mathrm{UR})$ for the genes in this module (see color key). Category name, ID and size (number category genes in gene list/category size) are given above each bar. For example it can be seen that the most significant Reactome pathway is 'Muscle contraction' and that it is one of the most down-regulated functional categories on average (blue color) and that 16 out of the 31 genes assigned to this category are differentially expressed between UE and UR cohorts only (i.e. not differentially expressed between TR and UR cohorts). Full results are provided in Additional file 2: Table S4

the training response (relative to the exercise response gene list). This training-specific gene list was also enriched for both signalling and neurological related categories (e.g. Signalling by GPCR:P $=2.5 \times 10^{-28}$, Neurological system process: $\left.P=2.3 \times 10^{-46}\right)$. The over- representation of the cellular component: Extracellular region was also highly significant $\left(P=1.90 \times 10^{-16}\right)$. The top twenty most significantly over-represented functional modules for this training-specific subset are given in Additional file 2: Table S6 and illustrated in Fig. 4. 


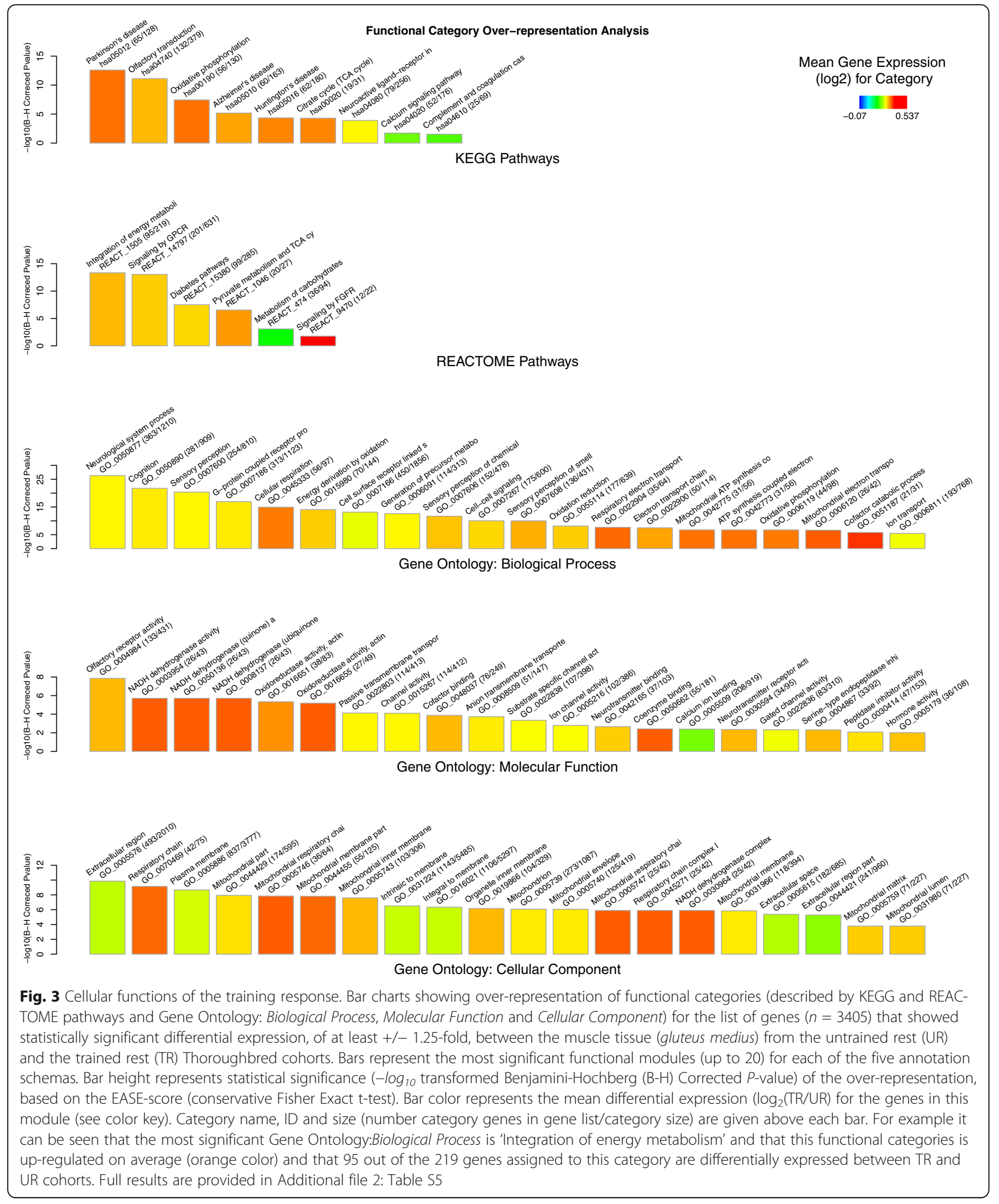

\section{Prediction of molecular interaction networks and their 're-wiring' in exercise and training}

The functional relevance of a gene or gene list in a context can only be fully appreciated by also examining how the genes, or their products, interact in that context. We therefore attempted to reconstruct context-specific dynamic PPI networks by integrating our exercise and training response gene lists and per cohort co-expression correlations with 
the global set of experimentally validated PPIs as described by EBI's IntAct Database. First, two dynamic networks were constructed to model how the genes associated with the exercise and training responses may 'interact' in each of the experimental cohorts. The set of genes (network nodes) in each dynamic interaction network was confined to those genes associated with that response. The putative interactions (network edges) were inferred from the gene expression correlations in each experimental cohort. The edges in each dynamic network had two states, the ground state, derived from the untrained rest (UR) cohort, and the active state, derived by either the exercise (UE) or trained (TR) cohort. Edges were also weighted with cohort specific correlation coefficients. To better model the putative contextual protein-protein interactions (PPIs) these correlation-based interaction networks were then rigorously pruned to remove edges with no supporting experimental PPI evidence, retrieved via EBI's IntAct database. An overview of this process is illustrated in Fig. 5, where output networks (i)(vi) reflect Figs. 6 and 7, and further details are given in Methods. The final putative dynamic PPI network for the exercise response contained 513 nodes (edgeless nodes were removed) and 514 edges in the untrained rest (UR) state, see Fig. 6(a), and 426 nodes and 390 edges in the exercise (UE) state, Fig. 6(b).

In the exercise network the top twenty most influential genes, in terms of both degree and betweeness were identified (see Table 2 and Fig. 6). We found that the GABARAPL1 gene (GABA type A receptor associated protein like 1), which was upregulated $(+1.49$-fold; $P=6.5 \times 10^{-12}$ ) in the exercise response, was the top hub and bottleneck in both the untrained rest (UR) and exercise (UE) network states. The edges of GABARAPL1 were partially 're-wired' with it gaining 14 edges, losing 22 and retaining 11 interactors in the exercise state relative to the rest state (see Additional file 1: Section 3.1). We also observed that relative influence of other hub and bottleneck genes appeared to change between network states. For example, DNAJB (dnaj hsp40 member B1), FN1 (fibronectin 1) and LIMA1 (LIM domain and actin binding 1) all had a greater influence in the exercise network state than the rest state as determined by both betweenness rank (FN1 from 12th to 2nd; LIMA1 from 73rd to 5th; $D N A J B$ from 174th to 3rd) and degree rank (FN1 from 5th to 2nd; LIMA1 from 40th to 3rd), see Table 2 and Fig. 6 . The additional edges gained by DNAJB1 in the exercise state included a new putative interaction with, GABARAPL1, which effectively boosts the network-wide influence (i.e. betweeness) of $D N A J B 1$ by proxy.

We then performed a systems level analysis of the exercise response dynamic network by applying Newman's fastgreedy community detection algorithm [45] to cluster the nodes in the network based only upon network topology. Twenty-eight non-trivial clusters (i.e. containing at least two genes) were detected in the untrained rest (UR) network state. The functional relevance of each of these clusters (de novo putative gene modules) was then evaluated by over-representation analysis and twentytwo clusters were found to be significantly enriched for known functional modules. The largest clusters were primarily enriched for the cellular components Contractile fibre (Cluster 1: 55 genes; $P=8.9 \times 10^{-07}$ ), Proton-transporting ATP synthase/ Mitochondrial respiratory chain complex $V$ (Cluster 4: 35 genes; $P=2.5 \times 10^{-10}$ ), NADH dehydrogenase/ Mitochondrial respiratory chain Complex $I$ (Cluster 6: 32 genes; $P=3.5 \times 10^{-35}$ ) and the biological process Pyruvate metabolism and TCA cycle (Cluster 7: 32 genes; $P=2.1 \times 10^{-18}$ ), see Fig. 6(a). To illustrate how these clusters are 're-wired' in the exercise state we then overlaid the rest state cluster membership onto the exercise network state, by applying the same node format (colour and shape) to common nodes, see Fig. 6(b).

We observed changes in the interactions of several clusters in the exercise state relative to the rest state. The cluster enriched for Pyruvate metabolism and TCA cycle (Cluster 7) for example, which contained 32 genes that were upregulated (mean:1.41-fold) in the exercise response, became disconnected in the exercise state and had far fewer internal interactions (seven edges) relative to the rest state (39 edges), see Fig. 6(a) and (b). The top hub within this cluster, SIRT4 (sirtulin 4) which was upregulated in the exercise response $\left(+1.69\right.$-fold; $\left.P=2.4 \times 10^{-14}\right)$, had 18 interactions in the rest state and only three interactions in the exercise state. The cluster enriched for Contractile fibre however (Cluster 1) became mostly downregulated (38/55 genes; mean: -1.2 -fold) in the exercise state relative to rest state. It also lost many putative interactions in the exercise state (22 edges) relative to the rest state (60 edges) and became fragmented. For example, phosphoinositide-3-kinase regulatory subunit 1 (PI3KR1), which was upregulated in the exercise state (1.7-fold; $\left.8.1 \times 10^{-12}\right)$, formed a new independent sub-cluster. This new cluster included new putative interactions with Cluster 31 members STAT3 (signal transducer and activator of transcription 3) and SP1 (Sp1 transcription factor) which were also upregulated in the exercise response 1.35-fold $\left(P=2.8 \times 10^{-12}\right)$ and 1.26 -fold $(P=0.0056)$ respectively (see Fig. 6b). Other clusters, however, were upregulated in a more coordinated manner in the exercise state (i.e. retained many intra-cluster edges). For example, the cluster enriched for $N A D H$ dehydrogenase/Mitochondrial respiratory chain complex I (Cluster 6), which is upregulated in the exercise response (mean:1.35-fold), retained 23 of its 52 rest state edges in the exercise state. Most these retained edges (20/23) were between genes that encode subunits of NADH dehydrogenase/ Respiratory chain complex $I$. The top hub in this cluster, in both 


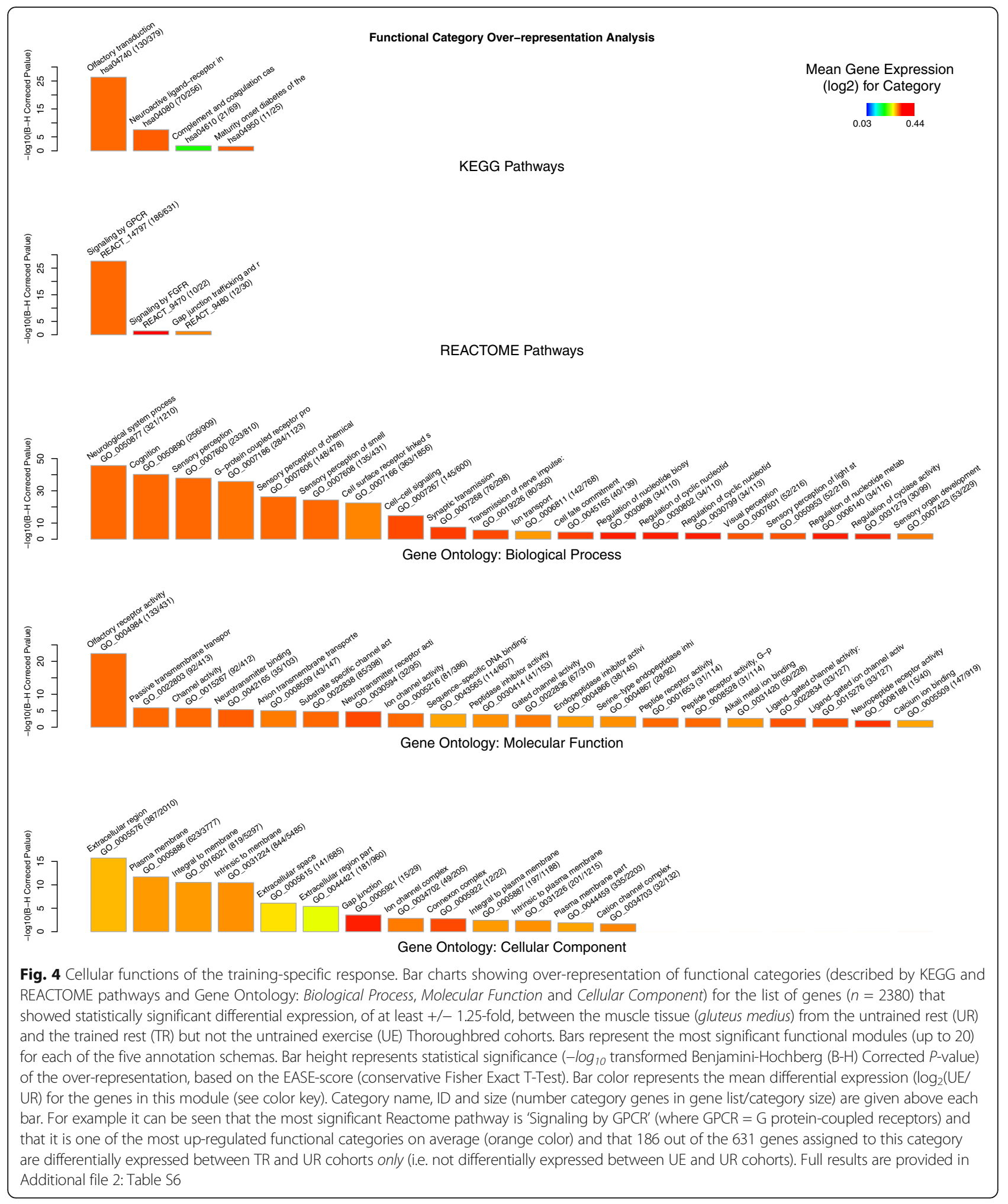

the rest (19 edges) and exercise state (16 edges), was NDUFA6 (NADH: ubiquinone oxidoreductase subunit A6). This cluster (Cluster 6) also became detached from the main network in the exercise state (along with 5 ATP synthase complex/ Respiratory chain complex $V$ genes from Custer 5), see Fig. 6(b), and its expression appears to be more independent from the rest of the network relative to rest state (where it previously had seven external edges). 


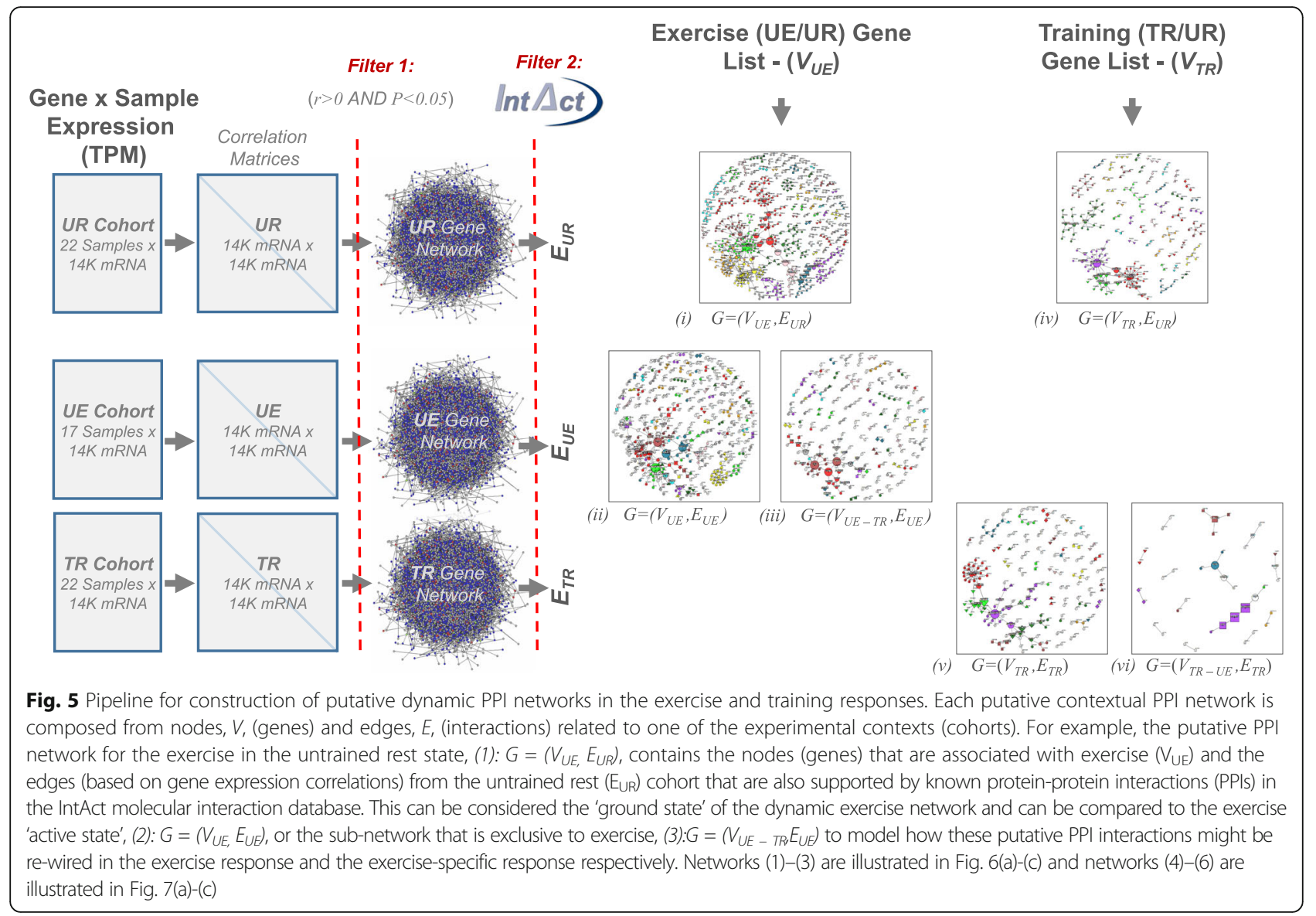

Some clusters became more central in the exercise state relative to the rest state, as determined by betweeness. For example, two genes in the cluster enriched for Apoptosis (Cluster 8), namely CDC37 (cell division cycle 37; -1.27-fold; $P=1.92 \times 10^{-07}$ ) and DNAJB (DnaJ Hsp4 member $B 1 ;+4.27$-fold; $\left.P=3.59 \times 10^{-14}\right)$, gained a greater network influence in the exercise state relative to the rest state, being promoted from 174th and 6th to 3rd and 4th respectively in rank based on betweenness (see Table 2). Additionally, two genes in the cluster enriched for Cytoskeleton (Cluster 5), FN1 (fibronectin 1; +1.29 -fold; $P=0.0018$ ) and LIMA1 (LIM domain and actin binding $1 ;+1.55$-fold; $P=9.7 \times 10^{-12}$ ), were also promoted from 8 th and 73 rd to 2 nd and 5 th respectively (see Table 2).

In the exercise-specific network (genes associated with the exercise but not the training response) the cluster enriched for Cytoskeleton (Cluster 5) was the largest upregulated cluster (see Fig. 6(c)). This upregulation also appears to be coordinated as it retained a majority of its intra-cluster interactions (14/26 edges). Genes in this cluster, FN1 and LIMA1, also became the top two bottlenecks in this exercise-specific network. FLNB (filamin B), which was also upregulated in exercise-specific response $(+1.66-$ fold; $P=1.1 \times 10^{-09}$ ), shared edges with both FN1 and LIMA1 and was the third most influential bottleneck in the exercise-specific response network.

In the training response putative dynamic PPI network, which was constructed as for the exercise response, there were 199 nodes and 186 edges in the untrained state (UR) and 188 nodes and 176 edges in the trained (TR) state (see Fig. 7). The top twenty most influential genes, determined by betweenness and degree, are given in Table 3 and highlighted in Fig. 7. As in the exercise response network, GABARAPL1 was the top bottleneck in the training response network both in the untrained state and trained state and was significantly upregulated $(+1.37$-fold; $P=0.0001)$ in response to training. GABARAPL1 was partially 're-wired' in the trained state (15 edges), gaining three and losing seven interactions relative to the untrained state (19 edges) of the network (see Additional file 1: Section 3.2). The highest degree node in both states was NDUFA6 (NADH ubiquinone oxidoreductase subunit A6), which was upregulated $(+1.36$-fold; $P=0.0007)$ in the training response and encodes a subunit of $N A D H$ dehydrogenase/ Mitochondrial respiratory complex I. Other top bottlenecks across both the untrained and trained network 

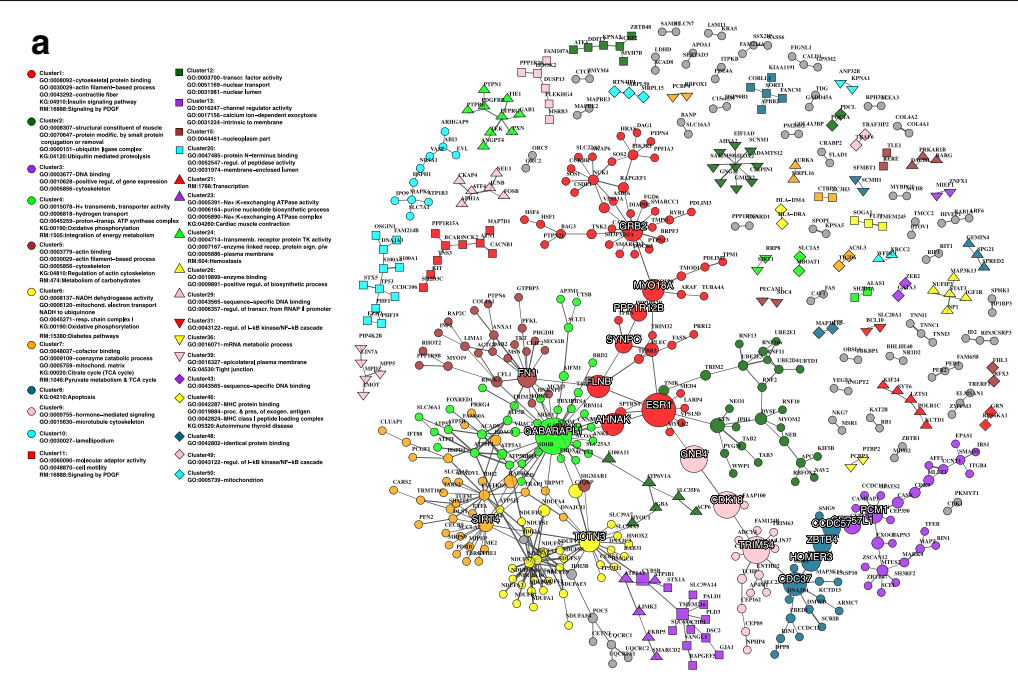

b
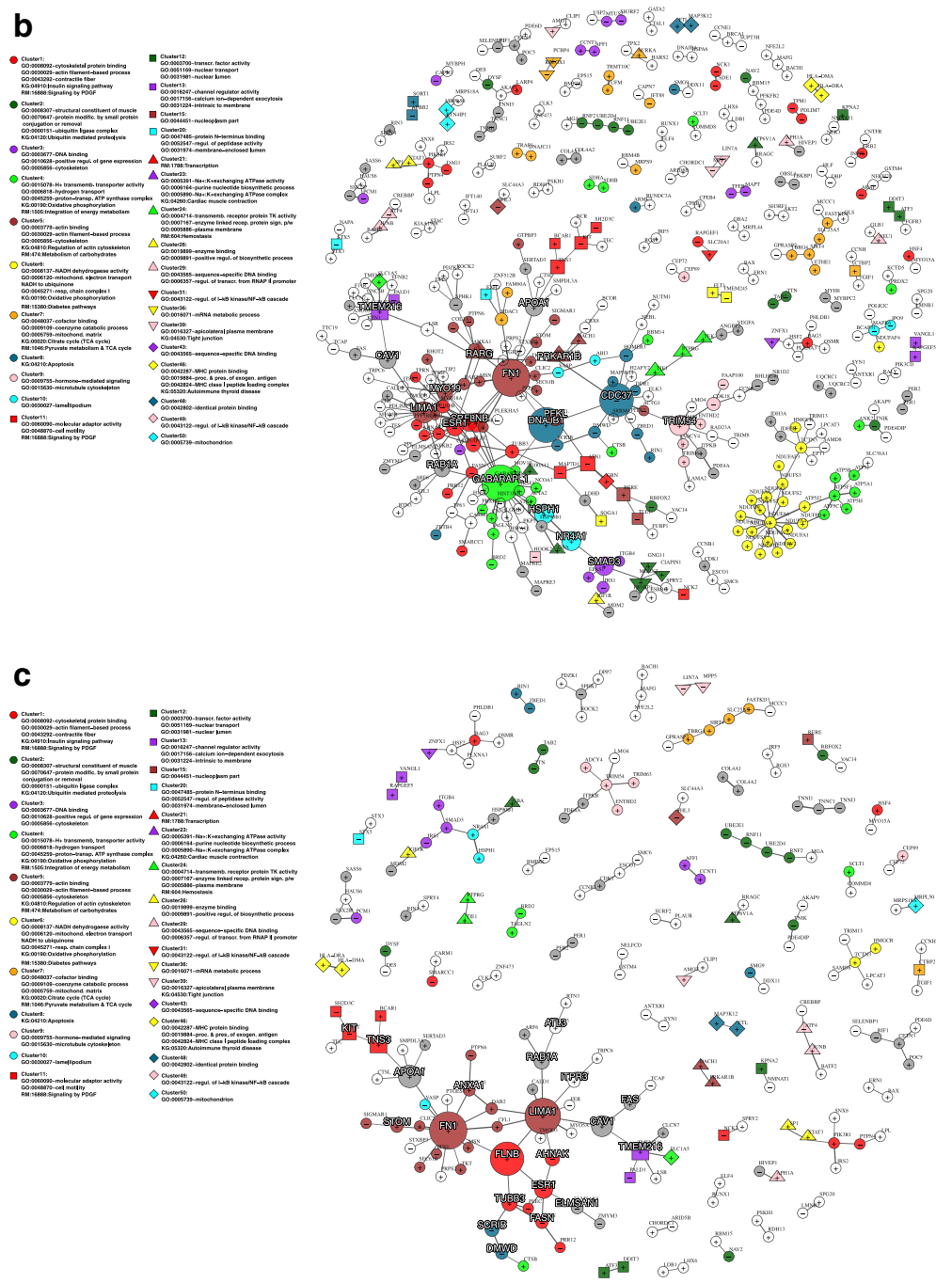

Fig. 6 (See legend on next page.) 


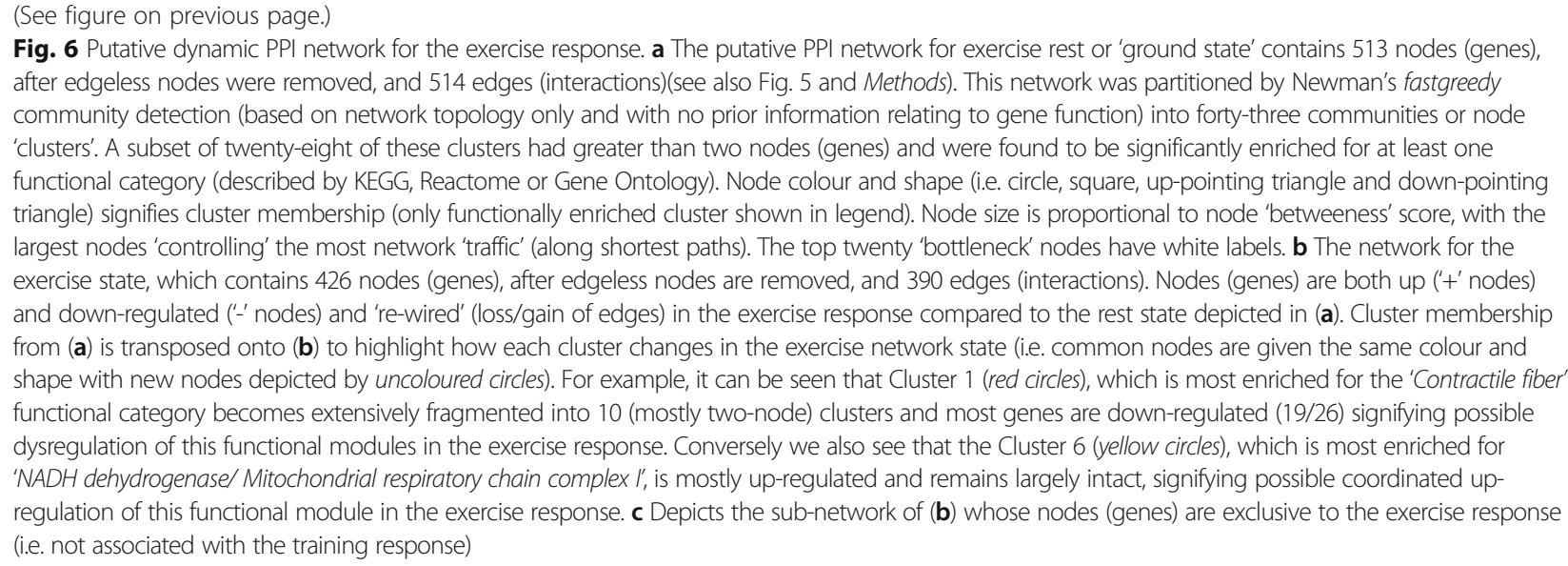

states included several genes that also encode subunits of this complex (NDUFA2, NDUFA4, NDUFA6, NDUFB3, NDUFV3) and ATP synthase complex/Respiratory chain complex $V$ (ATP5A1, ATP5B, ATP5C1, ATP5F1, ATP5J2). These genes were all significantly upregulated in the trained cohort (mean:1.38 fold; $P=0.0018$ ).

We observed that the network influence, as determined by hub or bottleneck status, of several genes changed in the trained state (TR) relative to the untrained network state (UR). SPTBN1 (spectrin beta, non-erythrocytic 1), which was upregulated in response to training $(+1.38$-fold; $P=0.0052$ ), was promoted in rank from 55th in the untrained state, to 3rd top bottleneck in the trained network state. SPTBN1 maintained its edge with GABARAPL1 but also appears to have undergone a 're-wiring' of other edges. For example, SPTBN1 gained edges with 'unconventional' myosins (as opposed to Class II myosins that are directly involved in muscle contraction [70]), MYO19 and $M Y O 18 A$, and an actin-associated protein gene SYNPO (synaptopodin). Conversely, the GRB2 (growth factor receptor bound protein 2) gene, which was downregulated $(-1.33$-fold, $P=0.0002)$ in the training response, became less influential in the trained (TR) state relative to the untrained network state, dropping from 5th to 49th place in the betweenness-based rankings.

Community analysis, performed as above, uncovered twenty-three clusters within the training response network untrained state. Of these, twelve non-trivial (more than two genes) clusters were significantly enriched for one or more known functional category (see Fig. 7(a)). Three of the clusters uncovered were interconnected (six edges) and were enriched for NADH dehydrogenase/ Mitochondrial respiratory complex I (Cluster 1), ATP synthase complex/ Mitochondrial respiratory complex $V$ (Cluster 34) and Pyruvate metabolism and TCA cycle (Cluster 3$)$. The genes in these clusters were mostly upregulated (i.e. $32 / 36,12 / 14$ and 10/20 genes respectively) in the training response (see Fig. 7(b)). These clusters also appeared to be upregulated in a coordinated manner due to the retention of many intra-cluster (internal) edges in the trained network state relative to the untrained state. The cluster enriched for NADH dehydrogenase/ Mitochondrial respiratory complex I (Cluster 1) retained 32/36 edges, the cluster enriched for ATP synthase complex/Respiratory complex $V$ (Cluster 34) retained 12/14 edges and the cluster enriched for Pyruvate metabolism and TCA cycle (Cluster 3) retained 10/ 20 edges (see Fig. 7(b)). Furthermore, these three clusters also retained 5/6 inter-cluster edges (between clusters) in the trained network state, implying coordination at the system level also. Another six-gene cluster, enriched for Signaling by VEGF/Focal adhesion (Cluster $6)$, was entirely upregulated (mean: 1.59-fold) in a coordinated manner (retained all intra-cluster edges) in the training state but remained independent from the other clusters (see Fig. 7(b)). In contrast to the above clusters, the cluster enriched for ERBB Signaling pathway/Signaling by PDGF (Cluster 2), which was the largest cluster (24 genes), became entirely downregulated (mean: -1.6fold) and dis-coordinated (retained only 6/25 intracluster edges) in the trained network state compared to the untrained network state (see Fig. 7(b)). Another 10gene cluster, which was enriched for Unfolded protein binding/ Postsynaptic density (Cluster 5), contained heatshock protein genes DNAJB1(DnaJ hsp40 member B1), $H S F 2 B P$ (heat shock transcription factor 2 binding protein) and co-chaperone genes CDC37 (cell division cycle 37) and CDC37L1 (cell division cycle like 1). This cluster became partially downregulated (five genes; -1.54 -fold) and upregulated (five genes; mean: +1.38 -fold) and 're-wired' (lost seven edges and gained one) in the training response.

The training-specific network (genes associated with the training response but not the exercise response) contained 25 putative interactions and little modular structure (only two modules with a diameter > 2) (see Fig. 7(c)). The largest contained six genes (including 

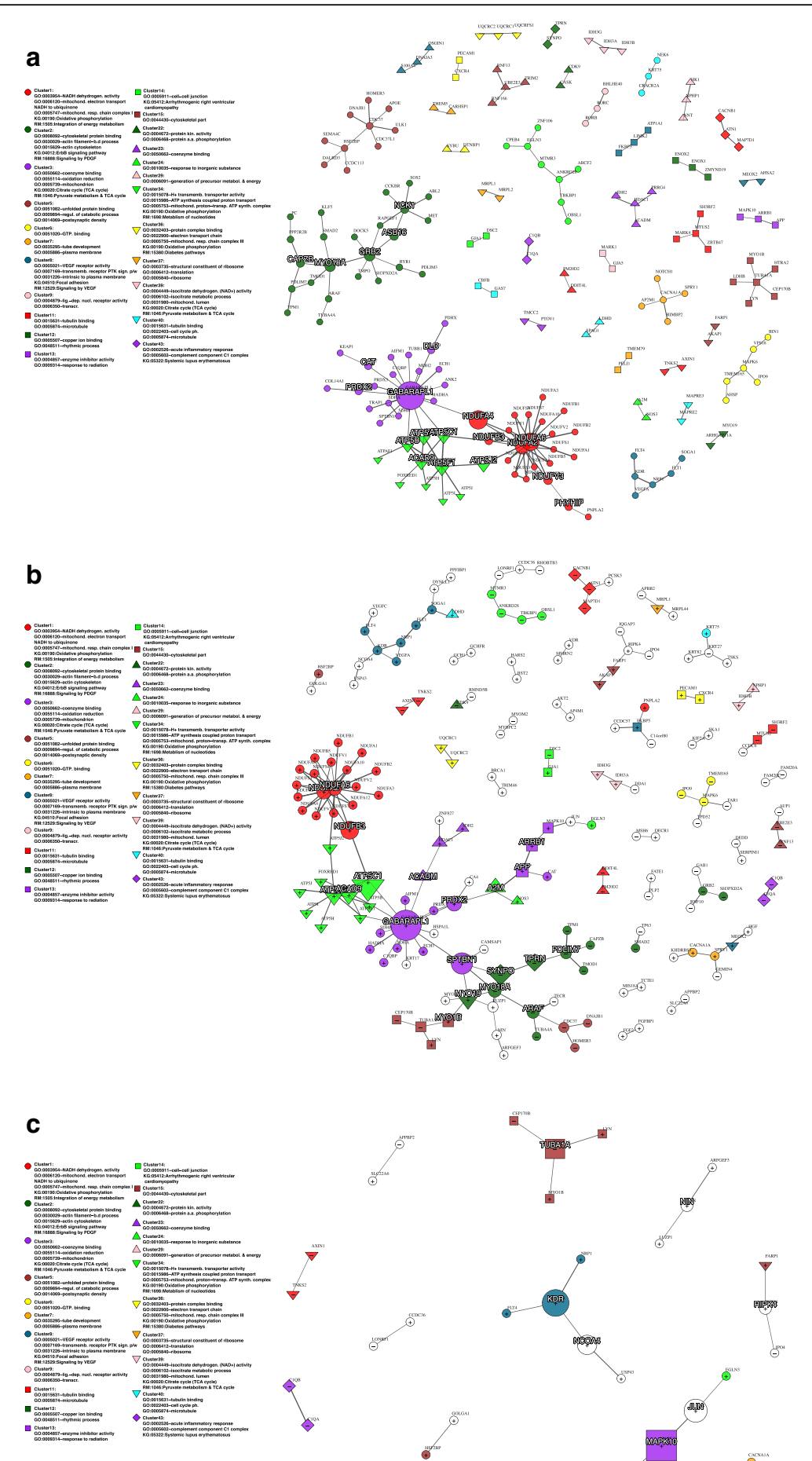

Fig. 7 (See legend on next page.) 
(See figure on previous page.)

Fig. 7 Putative dynamic PPI network for the training response. a The putative PPI network for untrained or 'ground state' contains 199 nodes (genes), after edgeless nodes were removed, and 186 edges (interactions)(see also Fig. 5 and Methods). This network was partitioned by Newman's fastgreedy community detection (based on network topology only and with no prior information relating to gene function) into forty-three communities or node 'clusters'. A subset of twenty-eight of these clusters had greater than two nodes (genes) and were found to be significantly enriched for at least one functional category (described by KEGG, Reactome or Gene Ontology). Node colour and shape (i.e. circle, square, up-pointing triangle and downpointing triangle) signifies cluster membership (only functionally enriched cluster shown in legend). Node size is proportional to node 'betweeness' score, with the largest nodes 'controlling' the most network 'traffic' (along shortest paths). The top twenty 'bottleneck' nodes have white labels. b The network for the trained state, which contains 188 nodes (genes), after edgeless nodes are removed, and 176 edges (interactions). Nodes (genes) are both up ('+' nodes) and down-regulated ('-' nodes) and 're-wired' (loss/gain of edges) in the training response compared to the untrained state depicted in (a). Cluster membership from (a) is transposed onto (b) to highlight how each cluster changes in the trained network state (i.e. common nodes are given the same colour and shape with new nodes depicted by uncoloured circles). For example, it can be seen that Cluster 2 (green circles), which is most enriched for the 'ErbB signaling pathway/Signaling by PDGF' functional category becomes fragmented into 4 clusters and all visible genes (edgeless nodes are not depicted) are down-regulated. Conversely we see that the Cluster 1 (red circles), which is most enriched for 'NADH dehydrogenase/ Mitochondrial respiratory chain complex l', is mostly up-regulated and remains largely intact, signifying possible coordinated up-regulation of this functional module in the training response. c Depicts the sub-network of (b) whose nodes (genes) are exclusive to the training response (i.e. not associated with the exercise response)

three genes from Cluster 13), which were all upregulated (mean: +1.3 -fold) in the training response, namely $A P P$ (amyloid beta precursor protein), ARRB1(arrestin beta 1), MAPK10 (mitogen-activated protein kinase 10), JUN (jun proto-oncogene AP-1 transcription factor subunit), EGLN3 (egl-9 family hypoxia inducible factor 3), and CAT (catalase) (Fig. 7(c)). Another module containing five genes was also upregulated in response to training (mean:1.49- fold) and contained part of the cluster enriched for Cytoskeleton (Cluster 8), namely KDR (kinase insert domain receptor, a.k.a. VEGFR-2), NRP1 (neuropilin 1) and FLT4 (fms related tyrosine kinase 4, a.k.a. VEGFR-3). This cluster also gained two additional interactions in the training response, $\mathrm{NCOA4}$ (nuclear receptor coactivator 4) and USP43 (ubiquitin specific peptidase 43), that were present in this training-specific sub-network.

Table 2 The top twenty hub (based on node degree, D) and bottleneck (based on node betweenness, B) genes in the Exercise Response network in the Untrained Rest (UR) and Untrained Exercise (UE) states

\begin{tabular}{|c|c|c|c|c|c|c|c|c|c|c|c|}
\hline \multicolumn{4}{|c|}{ Untrained Rest } & \multicolumn{4}{|c|}{ Untrained Exercise } & \multicolumn{4}{|c|}{ Unique to Untrained Exercise } \\
\hline Hubs & $D$ & Bottlenecks & $B$ & Hubs & $D$ & Bottlenecks & B & Hubs & $D$ & Bottlenecks & $B$ \\
\hline GABARAPL1 & 33 & GABARAPL1 & 20,436 & GABARAPL1 & 25 & GABARAPL1 & 6905 & FN1 & 15 & FN1 & 888 \\
\hline TCTN3 & 21 & ESR1 & 18,589 & FN1 & 19 & FN1 & 6881 & LIMA1 & 11 & LIMA1 & 826 \\
\hline NDUFA6 & 19 & TRIM54 & 13,553 & LIMA1 & 16 & DNAJB1 & 6327 & APOA1 & 5 & FLNB & 756 \\
\hline SIRT4 & 18 & GNB4 & 13,514 & NDUFA6 & 16 & CDC37 & 5983 & TRIM54 & 5 & APOA1 & 394 \\
\hline FN1 & 16 & CDK18 & 13,394 & CDC37 & 14 & LIMA1 & 2799 & TMEM216 & 5 & CAV1 & 346 \\
\hline GRB2 & 16 & CDC37 & 10,875 & MYO18A & 13 & TRIM54 & 2444 & ESR1 & 5 & ESR1 & 246 \\
\hline NDUFA2 & 15 & FLNB & 10,484 & TMEM216 & 11 & $\mathrm{HSPH} 1$ & 2338 & BAG3 & 4 & TMEM216 & 210 \\
\hline FASTKD3 & 15 & AHNAK & 10,125 & MYO19 & 10 & NR4A1 & 2210 & TCTN3 & 4 & TNS3 & 207 \\
\hline ESR1 & 10 & MYO18A & 9786 & SPTBN1 & 9 & FLNB & 2123 & TUBB3 & 4 & RAB1A & 158 \\
\hline TRIM54 & 9 & TCTN3 & 9292 & TRIM54 & 8 & SMAD3 & 2094 & FLNB & 4 & TUBB3 & 156 \\
\hline NCK1 & 9 & GRB2 & 8676 & ESR1 & 8 & APOA1 & 1759 & PIK3R1 & 4 & AHNAK & 120 \\
\hline MEOX2 & 8 & FN1 & 7852 & TCTN3 & 7 & CAV1 & 1707 & SMAD3 & 3 & $\mathrm{KIT}$ & 107 \\
\hline ATP5F1 & 8 & HOMER3 & 7598 & FLNB & 7 & RARG & 1691 & $\mathrm{KIT}$ & 3 & SCRIB & 106 \\
\hline CDC37 & 8 & ZBTB4 & 7364 & MEOX2 & 6 & TMEM216 & 1659 & TNS3 & 3 & FAS & 54 \\
\hline TMEM216 & 8 & CCDC57 & 7154 & ATP5F1 & 6 & ESR1 & 1603 & CAV1 & 3 & DMWD & 54 \\
\hline ACAD9 & 7 & CEP57L1 & 6650 & SYNPO & 6 & SPTBN1 & 1484 & CETN3 & 3 & ITPR3 & 54 \\
\hline PCM1 & 7 & PCM1 & 6593 & SMAD3 & 5 & RAB1A & 1322 & RAB1A & 3 & FASN & 54 \\
\hline $\mathrm{HDAC1}$ & 6 & SYNPO & 6545 & TUBB3 & 5 & PRKAR1B & 1300 & NR4A1 & 3 & ANXA1 & 54 \\
\hline NDUFS2 & 6 & PPP1R12B & 6084 & APOA1 & 5 & PFKL & 1162 & HSF2 & 3 & ELMSAN1 & 54 \\
\hline TTN & 6 & SIRT4 & 5093 & PLEC & 5 & MYO19 & 1095 & FASN & 3 & STOM & 54 \\
\hline
\end{tabular}

The top twenty hub and bottleneck genes are also provided for the sub-network that was unique to the Exercise Response (i.e. constructed from genes differentially expressed in response to exercise but not training) 
Table 3 The top twenty hub (based on node degree, D) and bottleneck (based on node betweenness, $B$ ) genes in the Exercise Response network in the Untrained Rest (UR) and Trained Exercise (TR) states

\begin{tabular}{|c|c|c|c|c|c|c|c|c|c|c|c|}
\hline \multicolumn{4}{|c|}{ Untrained Rest } & \multicolumn{4}{|l|}{ Trained Rest } & \multicolumn{4}{|c|}{ Unique to Trained Rest } \\
\hline Hubs & $D$ & Bottlenecks & $B$ & Hubs & $D$ & Bottlenecks & $B$ & Hubs & $D$ & Bottlenecks & B \\
\hline NDUFA6 & 20 & GABARAPL1 & 819 & NDUFA6 & 19 & GABARAPL1 & 2137 & KDR & 3 & MAPK10 & 6 \\
\hline GABARAPL1 & 19 & NDUFA4 & 459 & GABARAPL1 & 15 & ATP5C1 & 1631 & TUBA1A & 3 & ARRB1 & 6 \\
\hline NDUFA2 & 15 & NDUFA6 & 432 & NDUFA2 & 14 & SPTBN1 & 1135 & NCOA4 & 2 & $\mathrm{KDR}$ & 5 \\
\hline ATP5F1 & 8 & NDUFA2 & 320 & ATP5F1 & 8 & NDUFB3 & 1067 & NIN & 2 & JUN & 4 \\
\hline GRB2 & 7 & GRB2 & 177 & SPTBN1 & 5 & PRDX2 & 935 & JUN & 2 & APP & 4 \\
\hline CDC37 & 6 & MYO18A & 158 & ACAD9 & 5 & NDUFA2 & 933 & MAPK10 & 2 & NCOA4 & 3 \\
\hline ACAD9 & 6 & ATP5F1 & 125 & MYO19 & 5 & SYNPO & 842 & ARRB1 & 2 & TUBA1A & 3 \\
\hline NCK1 & 6 & NDUFV3 & 98 & MYO18A & 5 & $\mathrm{~A} 2 \mathrm{M}$ & 814 & APP & 2 & $\mathrm{NIN}$ & 1 \\
\hline TUBA1A & 5 & ATP5A1 & 88 & MAPK6 & 4 & NDUFA6 & 777 & RIPK4 & 2 & RIPK4 & 1 \\
\hline MYO18A & 5 & ASB16 & 85 & ARAF & 4 & MYO18A & 608 & TNKS2 & 1 & TNKS2 & 0 \\
\hline CACNA1A & 4 & ATP5B & 82 & LUZP1 & 4 & ARAF & 565 & IPO4 & 1 & IPO4 & 0 \\
\hline ATP5B & 4 & NCK1 & 82 & $\mathrm{KDR}$ & 4 & MYO19 & 549 & EGLN3 & 1 & EGLN3 & 0 \\
\hline HSF2BP & 4 & CAPZB & 78 & PRDX2 & 4 & APP & 509 & LUZP1 & 1 & LUZP1 & 0 \\
\hline ATP5C1 & 4 & ACAD9 & 76 & PDLIM7 & 4 & ACAD9 & 401 & $\mathrm{C} 1 \mathrm{QB}$ & 1 & $\mathrm{C} 1 \mathrm{QB}$ & 0 \\
\hline MAPK6 & 3 & ATP5J2 & 76 & SYNPO & 4 & ATP5F1 & 395 & C1QA & 1 & C1QA & 0 \\
\hline EGLN3 & 3 & ATP5C1 & 72 & ATP5C1 & 4 & TPRN & 323 & SPRY1 & 1 & SPRY1 & 0 \\
\hline $\mathrm{HDAC1}$ & 3 & NDUFB3 & 64 & $\mathrm{HDAC1}$ & 3 & PDLIM7 & 320 & FGF2 & 1 & FGF2 & 0 \\
\hline CAPZB & 3 & PHYHIP & 50 & SPRY1 & 3 & ACADM & 320 & FGFBP1 & 1 & FGFBP1 & 0 \\
\hline $\mathrm{KDR}$ & 3 & DLD & 50 & NDUFS2 & 3 & ARRB1 & 246 & CACNA1A & 1 & CACNA1A & 0 \\
\hline NDUFA4 & 3 & PRDX2 & 50 & CDC37 & 3 & MYO1B & 246 & DYNLL1 & 1 & DYNLL1 & 0 \\
\hline
\end{tabular}

The top twenty hub and bottleneck genes are also provided for the sub-network that was unique to the Training Response (i.e. constructed from genes differentially expressed in response to training but not exercise)

\section{Discussion}

Using a transcriptome-wide RNA-seq approach in a large cohort of active racehorses, we have for the first time generated a comprehensive set of genes that are differentially expressed in Thoroughbred skeletal muscle in response to both exercise and training. We have also developed a novel computational strategy to integrate these data with publicly available, experimentally validated PPIs in a bid to model the contextual interactome, its functional modules and how it may respond to exercise and training. We observed that approximately one third of genes that were diffientially expressed in response to a single bout of exercise accumulate with training leading to new baseline levels of gene expression, suggesting that increases in aerobic capacity may be brought about mainly through this mechanism. Therefore, consecutive bouts of exercise training seem to result in a priming of the skeletal muscle transcriptome for the demands of the next exercise bout. We have also demonstrated that this may further lead to an extensive 're-wiring' of the molecular interactome in both exercise and training and have identified key genes and functional modules that may be involved in controlling and mediating these responses.
In this study the training period was six months, therefore it is possible that some of the changes in gene expression attributed to training may be age-related. As this study was undertaken in collaboration with an active racing yard, which offers considerable advantages in terms of cohort size, consistent exercise/training regimen and consistent environmental factors, such as nutrition, certain controls, such as untrained mature horses were not possible to obtain at the same time point as the TR samples. However, training standards ensured that all time points in the study ( $>513$ days) were well beyond the end of the pubescent period for Thoroughbred horses ( $<450$ days) [71]. Furthermore, a small subset of the UR cohort $(N=4)$ was sampled after a two-week period of detraining, Detrained Rest (DR), and while there was some overlap among DE genes (28 genes), there was very little difference in terms of overall DE (144 genes after correction for multiple comparisons) between these two cohorts (See Additional file 1: Section 5 and Additional file 2: Table S7.)

Our initial functional over-representation analyses supported a central role for modules related to energy metabolism in both the exercise response and training response. Interestingly, several functional modules of this type, which 
were common to both, were more significantly associated with the training response than the exercise response in terms of module significance (see Fig. 1 vs. Fig. 3). For example, the exercise response was enriched for Integration of energy metabolism with a significance level of $P=4.97 \times 10$ ${ }^{-11}$; however, this increased to a significance level of $P=4.66 \times 10^{-14}$ in the training response. We also observed a similar trend for Pyruvate metabolism and TCA cycle (from $P=0.0204$ to $P=2.93 \times 10^{-07}$ ) and NADH dehydrogenase/Mitochondrial respiratory chain complex $I$ (from $P=0.0017$ to $\left.P=1.29 \times 10^{-06}\right)$. Furthermore, some functional modules related to energy metabolism were only found to be significantly over-represented in the training response gene list. These included the Citrate cycle $\left(P=5.12 \times 10^{-05}\right)$ and the respiratory chain component Proton-transporting ATP synthase / Respiratory chain complex $V(P=0.0007)$. These results support the hypothesis that trained skeletal muscle may exhibit an unstimulated transcriptome inherently primed to respond to the energy demands of exercise. More generally this information also provides support for the view that 'accumulative' changes play a role in the adaptive response.

There is also some evidence that epigenetic regulation may play a role in modulating these transcriptomic adaptations. For example, four of the five genes reported by Barrès and colleagues to be hypomethylated and expressed in human skeletal muscle in response to acute exercise, namely PPARD (peroxisome proliferator activated receptor delta), PPARGC1A (PPARG coactivator 1 alpha), PDK4 (pyruvate dehydrogenase kinase 4) and CS (citrate synthase), [72] were significantly upregulated either in the exercise response (PPARD:+1.97-fold; $P=1.2 \times 10^{-13}$, PPARGC1A:+10.17fold; $P=7.25 \times 10^{-26}, P D K 4:+2.17$-fold; $\left.P=0.0092\right)$ or the training response $\left(C S:+1.33\right.$-fold; $\left.P=1.40 \times 10^{-05}\right)$. In particular, we found that PPARGC1A, which was reported by Barrès and colleagues to be hypomethylated in response to acute exercise, was the 6th most significantly upregulated gene (10-fold, $\left.P=7.25 \times 10^{-26}\right)$ in the exercise response. PPARGC1A was also shown previously to be up-regulated at the protein level after high intensity exercise [73]. PPARGC1A directly links external physiological stimuli and mitochondrial biogenesis [74], regulates muscle fibre type [75] and is associated with endurance exercise [76]. It has also been shown to mediate the epigenetic regulation of insulin secretion [77] and regulate oxidative energy metabolism during exercise in equine skeletal muscle [30]. Our previous work has also supported the involvement of PPARGC1A in the adaptation of equine skeletal muscle to training [32, 78].

Another gene highlighted by Barrès et al., CS, which encodes citrate synthase that catalyses the synthesis of citrate from oxaloacetate and acetyl coenzyme A, was upregulated in the TR cohort. This may contribute to the trained cohort's constitutive priming for exercise. Indeed, increased
CS activity has previously been reported in trained human [79] and equine [80, 81] muscle and is a validated biomarker for skeletal muscle mitochondrial density and oxidative adaptation to a training [82].

We also observed that several functional modules uncovered by over-representational analysis were exclusively associated with either the exercise response or the training response. Only the exercise response, for example, was significantly enriched for functional modules related to muscle contraction. The largest module of this type was Contractile fibre $\left(P=6.35 \times 10^{-11}\right)$ and contained a majority $(44 / 56)$ of downregulated genes. This observation is in line with the previous finding of an inhibitory effect of NAD+/NADH ratio on MYOD1 regulated gene expression and differentiation [83]. NAD + /NADH ratio is generally found to increase in muscle cells post-exercise in animals but, curiously, not in humans $[84,85]$. We also observed that MYOD1, which is known as a 'master switch' of muscle cell differentiation [86], was significantly downregulated at the transcript level $(-1.9$-fold; $P=0.0008)$ but only in the training response, which suggests that it may form part of the adaptive response to training.

We also found that the Hemostasis pathway was exclusively significantly over-represented $\left(P=3.76 \times 10^{-05}\right)$ in the exercise response, it being mostly (46/69 genes) upregulated in exercise relative to rest cohort. This response included several upregulated growth factors, such as $E G F$, Epidermal Growth Factor, $\left(+1.73\right.$-fold; $\left.P=6.80 \times 10^{-08}\right)$, TGF-beta 2, transforming growth factor beta $2(+1.29$-fold; $P=0.0006)$ and VEGFA, vascular endothelial growth factor $A,\left(+2.33\right.$-fold, $\left.P=4.67 \times 10^{-19}\right)$, VEGFC, vascular endothelial growth factor $C$ (+1.79-fold, $\left.P=6.65 \times 10^{-10}\right)$ and VEGFD vascular endothelial growth factor $D,(+1.48-$ fold, $P=5.77 \times 10^{-09}$ ). This observed association of hemostasis with the exercise but not the training response gene list aligns with recent findings in human of a transient hypercoagulability state post-exercise, particularly in untrained individuals, that is largely reversed in trained individuals [87].

Conversely, the training response gene list was also exclusively enriched for certain functional modules, such as the pathways Signalling by GPCR $\left(P=1.22 \times 10^{-17}\right)$ and Neurological systems processes $\left(P=4.85 \times 10^{-27}\right)$, that were not associated with the exercise response. Gprotein-coupled receptors (GPCRs) mediate physiological responses to hormones, neurotransmitters and environmental stimulants [88]. The GPCR family also includes opioid receptors (ORs), discussed in further detail below in relation to the network analysis results.

Interestingly, we also observed over-representation of several disease related pathways in both our exercise and training associated gene lists. For example, both gene lists were enriched for Reactome's Diabetes pathways, 
which were more associated with the training response $\left(P=3.10 \times 10^{-08}\right)$ than the exercise response $\left(P=3.57 \times 10^{-06}\right)$. It is recognized that regular physical exercise, particularly aerobic and resistance exercise, has considerable health benefits for people with both type 1 and type 2 diabetes [89]. Our over-representation results also appear to provide some support for a relationship between our exercise phenotypes and diabetes and it would be interesting, in future studies, to investigate the extent of this mechanistic link. We also observed that several neurodegenerative disorders, namely Alzheimer's Disease (AD), Huntington's Disease (HD) and Parkinson's Disease (PD), were amongst the most significantly enriched KEGG categories associated with both the exercise response and the training response. The links between these neurodegenerative diseases and mitochondrial dysfunction have been well established [90] and the overrepresentation of these pathways may be due in part to the effect that intense exercise has on mitochondrial function in skeletal muscle. Interestingly, an early deficit of synaptic mitochondria and motor function has been associated with $\mathrm{AD}[90,91]$, physical exercise has been shown to be protective in both $\mathrm{AD}$ and $\mathrm{PD}$ [92] and increased mitochondrial biogenesis has recently been shown to improve symptoms in HD [93]. Our results also appear to reflect this mitochondrial-driven mechanistic relationship between exercise and this group of neurodegenerative disorders.

Standard over-representation analysis, as performed here, is based on well-established, carefully curated, functional annotations of genes and gene products (e.g. KEGG, Reactome, gene ontology). However, these resources are far from complete. For example $85 \%$ of Ensembl genes are not mapped to a KEGG pathway [34], and some genes may have additional undescribed roles [94]. For example, the kappa opioid receptor (KOR) signalling pathway, which we discuss below, was not described in sufficient detail by KEGG (only as part of the Neuro-active ligand receptor pathways) or fully labelled by Reactome (covers the $m u$ but not the kappa-OR, see [95]) to be identified by our above over-representation analysis. Furthermore, even well established and fully annotated pathways/complexes may behave in an unexpected manner in a particular context (e.g. cell type, species or experimental condition) [96]. In addition, due to the group-wise nature of over-representation analysis the chief drivers or regulators of biological processes may not always be apparent (nor are these, of course, necessarily highlighted by basic statistical association of expression gene). These limitations, however, may be somewhat overcome by employing a less supervised, network analysis-based approach.

When we constructed our putative contextual PPI networks for exercise and training we first identified the most influential genes in the various states as determined by hub and bottleneck status (see Tables 2 and 3). Interestingly, we found that some nodes appeared to gain influence in response to exercise and/or training relative to the untrained rest network state. In the exercise response network for example, FN1 (fibronectin) became more influential in the network after exercise relative to the untrained rest state (promoted from 11th to 2nd top bottleneck). FN1 is involved in cell adhesion, migration, growth and differentiation [97], and interestingly, has previously been used as an indicator of myofibre damage, such as may occur after intense exercise [98]. Another gene that appeared to become more influential in the post-exercise network state, LIMA1 (LIM Domain and actin binding 1), has been shown to stabilize the vascular capillary network in vitro [99]. This information, and the fact that neither FN1 nor LIMA1 expression was associated with the training response, might indicate involvement in muscle or microvascular recovery and repair following intense exercise. Continuing in this theme, the top differential bottleneck gene DNAJB1 (DnaJ Hsp40 Member B1), which was significantly $\left(P=3.6 \times 10^{-14}\right)$ upregulated $(>4$-fold) in response to exercise, has previously been found to be upregulated in skeletal muscle in response to heat shock [100]. Interestingly this is one of the few genes whose expression is also significantly reversed in the training response ( -1.6 -fold, $P=0.0005)$. This also supports previous studies which reported the significant upregulation (between 1.5 fold and 4.8-fold) of heat shock proteins both immediately after and $4 \mathrm{~h}$ post-exercise in Thoroughbred horses [27]. Together these data suggest that these genes may be involved in the stress response induced by intense exercise in the untrained cohort.

Several other genes, such as SIRT4 (sirtulin 4), GRB2 (growth factor receptor bound protein 2), GNB4 (G protein subunit beta 4) and CDK18 (cyclin dependent kinase 18), became less influential in the exercise network state relative to untrained rest network state (i.e. their hub/ bottleneck ranks were all down-graded after exercise). Although SIRT4 had far fewer edges in the exercise state relative to rest state ( 4 vs. 18 edges) it was highly significantly upregulated $\left(+1.69\right.$-fold; $\left.P=2.4 \times 10^{-14}\right)$ in the exercise response. This perhaps suggests a shift in function in response to exercise. SIRT4 has been shown to associate with mitochondria and to have 'strong and reproducible' ADP-ribosyltransferase activity [101]. SIRT4 is also required for protection against cell death caused by nutrient starvation via a role in the maintenance of mitochondrial NAD+ levels [102]. Interestingly, the only novel edge that was gained by SIRT4 in the exercise response was with SLC25A5 (solute carrier family 25 Member 5), which was also highly significantly upregulated in the exercise response only $\left(+3.2\right.$-fold; $\left.P=5.3 \times 10^{-21}\right)$ relative to untrained rest. SLC25A catalyses the exchange of mitochondrial ATP with cytoplasmic ADP across the 
mitochondrial inner membrane [103]. SIRT4 also retains an edge with ETHE1 (persulfide dioxygenase), which was also upregulated in the exercise response $(+1.3-$ fold; $P=9.5 \times 10^{-08}$ ). This mitochondrial matrix sulphur dioxygenase is involved in catabolizing hydrogen sulphide [104], a by-product of energy metabolism that inhibits mitochondrial functioning [105]. Together these data suggest that the re-wiring of SIRT4 interactions in the exercise state may be part of the exercise-induced stress response and may contribute to the maintenance of metabolic homeostasis in mitochondria.

In the trained network, we also observed evidence of a re-wiring of key hubs. SPTBN1 (spectrin beta chain nonerythrocytic 1), which was upregulated in the training response $(+1.38$-fold; $P=0.0052)$, appears to gain network influence in the trained network state (promoted in rank from 55th to 3rd top bottleneck). Functionally, SPTBN1 is involved in secretion, interacts with calmodulin in a calcium-dependent manner and has been isolated from post-synaptic density preparations [106]. Furthermore, SPTBN1 has previously been shown to be associated with skeletal muscle adaptation, specifically during the torpor phase of hibernation in mammals [107]. Metabolic adaptation and the prevention of muscle atrophy during hibernation induced starvation have also been previously linked to athletic endurance adaptions [108]. In further support of a mechanistic connection between these seemingly disparate physiological states, the reduced expression of the myostatin (MSTN) gene, referred to as the Thoroughbred 'speed gene' [13], has also been associated with hibernation in ground squirrels [109]. Similarly, another gene, PDK4 (pyruvate dehydrogenase kinase), has also been previously associated with hibernation in mammals as well as intense exercise in Thoroughbred horses [31, 110]. These data suggest perhaps that a similar biological mechanism may be involved both in the process of muscle growth during training and prevention of muscle wasting during prolonged inactivity and nutrient starvation.

The inferred contextual network may also provide some additional mechanistic insight into the activity of SPTBN1 in the context of skeletal muscle adaptations to training. In the trained network, the SPTBN1 bottleneck mediates the 'flow of information' between the module containing GABARAPL1 and the module containing unconventional myosins, MYO19 and MYO18A, and SYNPO (synaptopodin). MYO19, which was upregulated in both the exercise $\left(+1.5\right.$-fold; $\left.P=3.9 \times 10^{-08}\right)$ and trained $(+1.3$-fold; $P=0.0046)$ responses, is known to be involved in mitochondrial motility [111]. Furthermore, SYNPO, which was downregulated in both the exercise $(-1.66$-fold; $\left.P=6.7 \times 10^{-14}\right)$ and trained $\left(-1.59\right.$-fold; $\left.P=7.2 \times 10^{-05}\right)$ responses, is known to be part of the post-synaptic density [112]. ATP and $\mathrm{Ca}^{2+}$ flux is particularly important at the synapse, and mitochondria are usually present in presynaptic terminals [113]. Taken together the above 're-wiring' of SPTNB1 interactors may possibly reflect changes to the neuromuscular junction that occurs in response to exercise and training and may also suggests an increased influence of SPTNB1 in the training response.

Although we observed MSTN to be highly significantly downregulated in both the exercise $(-5.9$-fold; $\left.P=1.4 \times 10-^{19}\right)$ and training $\left(-6.4\right.$-fold; $\left.P=1.5 \times 10^{-06}\right)$ responses, it was absent from our putative contextual PPI networks as none of its contextual (correlationbased) edges were supported by experimentally validated PPIs. This may be partially explained by the stringency of our protocol (e.g. the exclusion of inverse co-expression correlations, inclusion only of PPIs supported by IMEX standard) and also lack of recorded interaction evidences for MSTN, due in part to that fact the MSTN ligand generally interacts with membrane-bound proteins, which are under-represented in PPI databases [114]. However, by examining the larger non-validated correlation-based networks we observed some evidence of a change in MSTNrelated activity in the exercise and training responses. We saw a reduction in MSTN edges in both the exercise and training network states relative to the untrained rest state. Also, approximately 20\% (43/231) of exercise-induced MSTN edges were also observed in the trained state, suggesting that there may be some sustained adaptation of the MSTN-related signalling network in the trained cohort, although, of course, further support is required to substantiate this. Interestingly however, this list of 43 genes includes PVALB (parvalbumin), which was the most downregulated gene in both the exercise response $\left(-25.43\right.$-fold; $\left.P=3.5 \times 10^{-20}\right)$ and the training response (-33.27-fold; $\left.P=7.8 \times 10^{-10}\right)$. PVALB is involved in calcium sequestration after muscle contraction to support muscle relaxation and a recognized fast-twitch phenotype gene [115].It has also been found that double-knockout $\mathrm{PV}-/-$ mice have prolonged muscle relaxation time, due to the maintenance of muscle $\mathrm{Ca}^{2+}$ levels, but also generate $\sim 40 \%$ more force per contraction than $\mathrm{PV}+/-$ and WT animals [116].

By far the most influential gene, as determined by hub and bottleneck analysis, across both the exercise and training response networks was GABARAPL1 (GABA type $A$ receptor associated protein like 1$)$, where it ranked as top bottleneck across all network states (Tables 2 and 3). We found GABARAPL1 to be highly significantly upregulated $\left(+1.49\right.$-fold; $\left.P=6.5 \times 10^{-12}\right)$ in response to exercise and significantly upregulated $(+1.37$-fold; $P=0.0001)$ in the trained cohort compared to the untrained cohort.

The product of GABARAPL1 is a ubiquitin-like modifier associated with the autophagosome and known to cause an increase in cell-surface expression of the $G$ 
protein-coupled receptor (GPCR) kappa type opioid receptor (KOR), through facilitating anterograde trafficking of the receptor [117]. KOR is the product of the OPRK1 gene. In our data OPRK1 mRNA expression showed no association with the exercise response, however it was significantly upregulated $(+1.74$-fold; $P=0.0075)$ in the training response. Taken together this information supports the model of an initial post-translational upregulation of the KOR cell-surface expression by GABARAPL1 in response to exercise and then, upon adaption, a transcriptional upregulation in the trained cohort. However, the downstream effect of the putative upregulation of the KOR in the context of exercise and training still needs to be clarified. KOR mediated signal transduction is known to stimulate several kinase cascades depending upon the specific activating ligand [118]. For example, dynorphin activation of KOR has been shown to be involved in the stress response (e.g. to stressors such as forced swim) in mice [119]. This same ligand-receptor interaction has also been associated with pro-addictive behaviour [120]. Further work is needed in this context, but we hypothesize that the regulation of aversive/motivational behaviour by KORs may play a role in the adaptation of the organism to regular and sustained bouts of intense exercise.

We can also attempt to extract additional mechanistic and functional insight into GABARAPL1 in the context of exercise and training from community analysis. GABARPL1 is a member of the exercise network cluster that is significantly enriched $\left(P=2.56 \times 10^{-10}\right)$ for the Proton-transporting ATP synthase/Mitochondrial respiratory chain complex $V$ (MRC complex $V)$. This cluster also has four putative interactions with the cluster that is significantly enriched $\left(P=9.69 \times 10^{-38}\right)$ for $N A D H$ dehydrogenase/ Mitochondrial respiratory chain complex I (MRC complex I) (see Fig. 6(a)). In the exercise response, we observed a general upregulation of the genes in both clusters and a general disturbance in the rest of the network (see Fig. 6(b)). These clusters became 're-wired' but remained largely intact except for a major de-coupling of the MRC complex I-enriched cluster (along with genes encoding members of MRC complex $V$ ) from the rest of the network in the exercise state (see Fig. 6(b)). We observed that correspondingly enriched clusters (i.e. also enriched for MRC complexes I and V) were also present and upregulated in the trained state network (see Fig. 7(b)). However, in this case, the upregulation of these clusters was better coordinated with the rest of the network and was not accompanied by the similar de-coupling. Interestingly, this apparent 'de-coupling' of $M R C$ complexes $I$ and $V$ during exercise, may be a reflection of the recognized physiological response to oxidative stress known as uncoupling and mediated by UCP3 in skeletal muscle [23]. This is also supported by the highly significant upregulation of UCP3 $\left(+4.8\right.$-fold; $\left.P=7.6 \times 10^{-22}\right)$ in the exercise response. Interestingly, we did not see the differential expression of $U C P 3$ in the training response (although we did observe a significant increase $(+1.27$-fold; $P=0.0007)$ of $U C P 1$ and a decrease of $U C P 2(+1.47$-fold; $P=0.0175)$ ).

It has been suggested that NADH dehydrogenase/ Mitochondrial respiratory chain complex $I$ activity is among the major rate-controlling steps in mitochondrial oxidative phosphorylation [121], thus its coordinated upregulation in the training response may represent a significant adaption for the overall process of oxidative phosphorylation. The central position of GABARAPL1 in both the exercise and trained networks may suggest a driver/regulatory role in these responses, perhaps mediated in part via its control of kappa OR cell-surface expression, and downstream effects may include the altered regulation of the components of the mitochondrial respiratory chain. Previous studies have shown that the knock-down of GABRAPL1 leads to many cellular bioenergetic changes, including increased basal oxygen consumption rate, increased intracellular ATP (possibly due to OR-mediated dysregulation of adenylyl cyclinase/ cAMP signalling), increased total glutathione, and an accumulation of damaged mitochondria [117]. It has also recently been reported that the dysregulation of autophagy, in which GABARAPL1 has a central role, is linked to several neuromuscular disorders in human [122]. In the context of exercise and training, autophagy has already been shown to be activated by high-intensity, but not low-intensity, exercise in human [123] and to be involved in the maintenance of muscle mass in $\operatorname{Atg} 7$ (autophagy related 7) knock-out mice [124]. It has also been proposed that autophagosomal proteins, of which GABARPL1 is one, may act as sensors for oxidative stress (via redox signalling) and mediate cross-talk between this and other cellular processes such as mitochondrial turnover [125]. This latter relationship, in particular, would explain the tight coupling of GABARAPL1, NADH dehydrogenase/ Mitochondrial respiratory chain complex I and Proton-transporting ATP synthase/ Mitochondrial respiratory chain complex $V$ clusters that we observed in the exercise (rest state) and training networks. There is already some experimental evidence to support a role for autophagy in muscle cell homeostasis during exercise [126, 127]. Furthermore, there is support for GABARAPL1 being directly involved in mediating the autophagic energy stress response in heart muscle, involving both mitophagy and glycophagy, via its interaction starch binding domain 1 (STBD1) protein [128]. Our results indicate that GABARAPL1 may play a central role in the adaption to oxidative stress induced by intense exercise and may also play a key role in the adaptive response to training, perhaps in part via a role in the recycling and upregulation of mitochondria via mitophagy. 
The training response network also contains evidence of ancillary adaptations. For example, a small three-gene module, containing APP (amyloid beta precursor protein), ARRB1(arrestin beta 1) and MAPK10 (mitogen-activated protein kinase 10), that is enriched for Enzyme inhibitor activity/Response to radiation was initially disconnected from the main network in the untrained rest state (see Fig. 7(a)). This module however was upregulated and 're-wired' in the trained network state (see Fig. 7(b)), gaining several second-degree connections with GABRAPL1. Importantly, this module was also unique to the trained network (i.e. genes not associated with the exercise response) (see Fig. 7(c)). Arrestins such as ARRB1 are known to promote agonist-mediated desensitization of G protein-coupled receptors [129] and kappa and delta OR-mediated $G$ protein activation has been shown to be attenuated by over-expression of ARRB1 in fibroblast-like cell lines [130]. This module is also extended with the addition of several genes also unique to the training response (see Fig. 7(c)), namely CAT (catalase) and EGLN3 (egl-9 family hypoxia-inducible factor 3) and JUN (Jun proto-oncogene, AP-1 transcription factor subunit), which were also upregulated at a transcriptional level in the trained cohort. Catalase helps protect the cell against hydrogen peroxide mediated cytotoxicity especially when levels of pyruvate are low [131]. EGLN3 is one of the most important isoenzymes in modulating the hypoxic response [132] and has also been reported to regulate myogenin expression and skeletal muscle differentiation. Interestingly, JUN is known to be activated by ORs [133] and has been found to be involved in oxidative stress-mediated suppression of insulin gene expression [134]. Another six-gene cluster in the training response network, that was upregulated in a coordinated manner in response to training, contained genes that encode ligands and receptors of the VEGF signalling pathway. Several of these, namely $K D R$, $N R P 1$ and FLT4, were exclusive to the training response. In support of this, skeletal myofiber VEGFA has been found to be essential for the exercise training response in mice [135]. These data suggest that there may be coordinated upregulation of several genes specific to the training response that may control ancillary processes in the adaptive response to training in the skeletal muscle of the Thoroughbred.

\section{Conclusion}

Functional category over-representation analysis is a commonly used and valuable aid to the biological interpretation of gene/protein lists. However, this analysis may be improved with additional information (e.g. molecular interactions inferred from gene expression) derived from the experimental context in question (e.g. post-exercise skeletal muscle) and a contribution from unsupervised methods (e.g. community analysis) in defining functional classes. The absence of these considerations risks underexploiting rich high-throughput datasets and overlooking novel information that might help to expand knowledge of biological systems. As this is the first demonstration of this computational method, we have applied a stringent implementation to maximize quality over scale; however, in the future more refined implementations could be developed. Our results clearly demonstrate that network analysis and its capacity to predict influential nodes is very useful for highlighting putative control points. For example, our hypothesis that GABARAPL1 and autophagy may play a central role in the response to exercise and training was generated solely from this network analysis approach. Standard over-representation analysis enabled some insight into downstream effects (e.g. enrichment for energy metabolism, neurodegenerative disease etc.) but did not highlight the autophagy process nor point to a putative driver gene. Neither was this indicated by differential gene expression analysis (GABARAPL1 was outside the top 250 genes most significantly associated with exercise or training). Therefore, we have demonstrated the benefits of a novel network-based approach, which may serve as a model for future transcriptomics-based investigations into the molecular dynamics of biological systems in health and disease.

Taken together our results, both from the overrepresentation analysis and our novel network analysis approach, support the involvement of mitochondrial respiratory complexes I (NADH dehydrogenase) and V (ATP Synthase) in both the exercise and training responses. As expected, the exercise response in the untrained cohort appeared to include elements of a stress response but also included the downregulation of genes involved in myogenic differentiation. Neither of these features were observed in the trained cohort, although we cannot preclude them from a 'trained' exercise response based on the current data. It is also noteworthy that there is some limited support for the constitutive downregulation of heatshock proteins in the trained cohort. However, we did observe strong indications of the constitutive and coordinated upregulation of the mitochondrial respiratory complexes I and $\mathrm{V}$ in the trained cohort, which may suggest that the skeletal muscle has adapted to regular bouts of intense-exercise in this manner, presumably improving the efficiency of oxidative phosphorylation. There is also the suggestion from our network analysis that this upregulation of mitochondrial complexes, and indeed mitochondria, in response to exercise and especially training may be controlled in part by autophagy (and perhaps mitophagy) via GABARAPL1. Our results also suggested a connection between GABAR$A P L 1$ and the stimulation of/response to GPCR, especially via the opioid receptor, signalling, in both exercise and training. The network analysis also suggested that the 
SPTBN1 gene may play an important role in the adaptive response and that this response may include re-wiring of its interactions at the post-synaptic neuromuscular junction. Somewhat unexpected was the strong overlap between the exercise and training responses and pathways linked to several neurodegenerative diseases. However, there seems to be a strong body of evidence for an established role for mitochondrial dysfunction and autophagy in neurodegenerative disease. This also supports our hypothesis of the central involvement of these processes in both the exercise and training response in skeletal muscle. The involvement of authophagy and mitochondrial activity at the synaptic junction in both neurodegenerative disease and athletic adaptation seems to be a common theme, but further validation of this largely in silico generated hypothesis is required. In recent years autophagy and its role in many cellular processes and diseases [136] has garnered much interest and already autophagy-enhancing therapeutics have been proposed [137]. This study has provided further support for this process in relation to exercise (especially intense exercise), provided novel evidence of its central involvement in the adaptation of skeletal muscle to training and identified the autophagosomal gene GABARAPL1 as a potential regulatory target.

\section{Additional files}

Additional file 1: Supplementary Information Main Document. (DOCX $110 \mathrm{~kb})$

Additional file 2: Supplementary Tables. (XLS 2823 kb)

\begin{abstract}
Abbreviations
AD: Alzheimer's Disease; AMP: Adenosine Monophosphate; ATP: Adenosine Triphosphate; BP: Gene Ontology Biological Process; CC: Gene Ontology Cellular Component; DAVID: he Database for Annotation, Visualization and Integrated Discovery; GO: Gene Ontology; HD: Huntington's Disease; KEGG: Kyoto Encyclopaedia of Genes and Genomes; KOR: kappa Opioid Receptor; MF: Gene Ontology Molecular Function; MHC: Myosin Heavy Chain; miRNA: micro-RNA; mRNA: messenger RNA; MSTN: Myostatin; MYOD/ 1: Myogenic differentiation 1; NADP: Nicotinamide adenine dinucleotide phosphate; OR: Opioid Receptor; PD: Parkinson's Disease; PPI: Protein-protein interaction; PSICQUIC: Protein Standard Initiative Common Query Interface; TCA: The Citric Acid (Kreb's) Cycle; TR: Trained rest cohort; UE: Untrained exercise cohort; UR: Untrained rest cohort; WD: Work Day
\end{abstract}

\section{Acknowledgements}

We would like to acknowledge the access to horses facilitated by trainer JS Bolger and assistance from B O'Connor, P O'Donovan and yard staff at Glebe House Stables, Coolcullen, Co. Kilkenny, Ireland.

\section{Funding}

This publication has emanated from research conducted with the financial support of Science Foundation Ireland under grant number 11/PI/1166.

\section{Availability of data and materials}

The data sets generated for this paper are included within the article and the associated additional files. The RNA-seq aligned sequence files (BAM format) are available from the EBI ArrayExpress (https://www.ebi.ac.uk/arrayexpress) under accession number E-MTAB-5447.

\section{Ethics approval}

University College Dublin Animal Research Ethics Committee approval, a Department of Health License (B100/3525) and explicit owner/trainer informed consent were obtained for the use of all horses and procedures in this study.

\section{Authors' contributions}

KB performed the downstream bioinformatics analysis, interpreted results and wrote the manuscript. BAM contributed to study design, performed RNA-seq data analysis and contributed to writing the manuscript. GF was involved in sample collection, prepared the samples and contributed to RNAseq analysis. PAM contributed to study design and implemented an RNA-seq data pre-processing pipeline. CLM was involved in phenotyping and sample collection. KFG was involved in phenotyping and sample collection. DEM contributed to study design and manuscript preparation. LMK designed the study, coordinated phenotyping and sample collection and contributed to manuscript preparation. EWH conceived and designed the study, was involved in phenotyping and sample collection and contributed to results interpretation and writing the manuscript. All authors read and approved the final manuscript.

\section{Consent for publication}

All authors read and approved the final manuscript

\section{Competing interests}

None of the authors has any financial or personal relationships that could inappropriately influence or bias the content of the paper. EWH and DEM are shareholders in Plusvital Ltd., an equine nutrition and genetic testing company. Plusvital Ltd. has been granted a license for commercial use of data contained within patent applications: United States Provisional Serial Number 61/136553 and Irish patent application number 2008/0735, Patent Cooperation Treaty filing: A method for predicting athletic performance potential, September 7, 2009. EWH, DEM and LMK are named on the applications. The patent contents are not related to this manuscript. Plusvital Ltd. had no part in the research in the manuscript.

\section{Publisher's Note}

Springer Nature remains neutral with regard to jurisdictional claims in published maps and institutional affiliations.

\section{Author details}

${ }^{1}$ UCD School of Agriculture and Food Science, University College Dublin, Belfield D04 V1W8, Ireland. ' UCD Conway Institute of Biomolecular and Biomedical Research, University College Dublin, Belfield D04 V1W8, Ireland. ${ }^{3}$ UCD School of Veterinary Medicine, University College Dublin, Belfield D04 V1W8, Ireland.

Received: 1 February 2017 Accepted: 2 August 2017

Published online: 09 August 2017

\section{References}

1. Hinchcliff KW, Kaneps AJ, Geor RJ. Equine exercise physiology: the science of exercise in the athletic horse. New York. Edinburgh: Saunders/Elsevier; 2008.

2. Dingboom EGDG, Enzerink E, van Oudheusden HC, Weijs WA. Postnatal muscle fibre composition of the gluteus medius muscle of Dutch Warmblood foals; maturation and the influence of exercise. Equine Vet J. 1999;31(Suppl):95-100.

3. Rivero JLL, Talmadge RJ, Edgerton VR. Correlation between myofibrillar ATPase activity and myosin heavy chain composition in equine skeletal muscle and the influence of training. Anat Rec. 1996;246(2):195-207.

4. Coffey VG, Hawley JA. The molecular bases of training adaptation. Sports Med. 2007;37(9):737-63.

5. Serrano A, Quiroz-Rothe E, Rivero J-L. Early and long-term changes of equine skeletal muscle in response to endurance training and detraining. Pflugers Arch. 2000;441(2-3):263-74.

6. Rivero J, Ruz A, MARTI-KORFF S, Lindner A. Contribution of exercise intensity and duration to training-linked myosin transitions in thoroughbreds. Equine Vet J. 2006;38(S36):311-5. 
7. Eto D, Yamano S, Mukai K, Sugiura T, Nasu T, Tokuriki M, Miyata H. Effect of high intensity training on anaerobic capacity of middle gluteal muscle in thoroughbred horses. Res Vet Sci. 2004;76(2):139-44.

8. Lacombe VA, Hinchcliff KW, Taylor LE. Interactions of substrate availability, exercise performance, and nutrition with muscle glycogen metabolism in horses. J Am Vet Med Assoc. 2003;223(11):1576-85.

9. Derave W, Everaert I, Beeckman S, Baguet A. Muscle carnosine metabolism and $\beta$-alanine supplementation in relation to exercise and training. Sports Med. 2010;40(3):247-63.

10. Avellini L, Chiaradia E, Gaiti A. Effect of exercise training, selenium and vitamin E on some free radical scavengers in horses (Equus Caballus). Comp Biochem Physiol B: Biochem Mol Biol. 1999;123(2):147-54.

11. Barrey E, Valette J, Jouglin M, Blouin C, Langlois B. Heritability of percentage of fast myosin heavy chains in skeletal muscles and relationship with performance. Equine Vet J. 1999:31(S30):289-92.

12. Petersen JL, Valberg SJ, Mickelson JR, McCue ME. Haplotype diversity in the equine myostatin gene with focus on variants associated with race distance propensity and muscle fiber type proportions. Anim Genet. 2014;45(6):827-35.

13. Hill EW, McGivney BA, Gu J, Whiston R, MacHugh DE. A genome-wide SNPassociation study confirms a sequence variant (g. 66493737 C $>$ T) in the equine myostatin (MSTN) gene as the most powerful predictor of optimum racing distance for Thoroughbred racehorses. BMC Genomics. 2010;11(1):1.

14. Ferrell RE, Conte V, Lawrence EC, Roth SM, Hagberg JM, Hurley BF. Frequent sequence variation in the human myostatin (GDF8) gene as a marker for analysis of muscle-related phenotypes. Genomics. 1999;62(2):203-7.

15. Hill EW, Gu J, Eivers SS, Fonseca RG, McGivney BA, Govindarajan P, Orr N, Katz LM, MacHugh D. A sequence polymorphism in MSTN predicts sprinting ability and racing stamina in thoroughbred horses. PLoS One. 2010;5(1):e8645.

16. Binns M, Boehler D, Lambert D. Identification of the myostatin locus (MSTN) as having a major effect on optimum racing distance in the thoroughbred horse in the USA. Anim Genet. 2010;41(s2):154-8.

17. Tozaki T, Miyake T, Kakoi H, Gawahara H, Sugita S, Hasegawa T, Ishida N, Hirota K, Nakano Y. A genome-wide association study for racing performances in thoroughbreds clarifies a candidate region near the MSTN gene. Anim Genet. 2010;41(s2):28-35.

18. Hill EW, Ryan DP, MacHugh DE. Horses for courses: a DNA-based test for race distance aptitude in thoroughbred racehorses. Recent Pat DNA Gene Sequences, 2012. 6(3):203-8.

19. McGivney BA, Browne JA, Fonseca RG, Katz LM, MacHugh DE, Whiston R, Hill EW. MSTN genotypes in thoroughbred horses influence skeletal muscle gene expression and racetrack performance. Anim Genet. 2012;43(6):810-2.

20. Petersen JL, Mickelson JR, Rendahl AK, Valberg SJ, Andersson LS, Axelsson J, Bailey E, Bannasch D, Binns MM, Borges AS. Genome-wide analysis reveals selection for important traits in domestic horse breeds. PLOS Genet. 2013:9(1):e1003211.

21. Tyler CM, Golland LC, Evans DL, Hodgson D, Rose RJ. Skeletal muscle adaptations to prolonged training, overtraining and detraining in horses. Pflugers Arch. 1998;436(3):391-7.

22. Geor R, McCutcheon L, Hinchcliff K, Sams R. Training-induced alterations in glucose metabolism during moderate-intensity exercise. Equine Vet J. 2002; 34(S34):22-8.

23. Pilegaard H, Ordway GA, Saltin B, Neufer PD. Transcriptional regulation of gene expression in human skeletal muscle during recovery from exercise. Am J Physiol-Endocrinol Metab. 2000;279(4):E806-14.

24. Flück $\mathrm{M}$, Hoppeler $\mathrm{H}$. Molecular basis of skeletal muscle plasticity-from gene to form and function. In: Rev Physiol Biochem Pharmacol. Springer Berlin Heidelberg; 2003. p. 159-216.

25. Perry CG, Lally J, Holloway GP, Heigenhauser GJ, Bonen A, Spriet LL. Repeated transient mRNA bursts precede increases in transcriptional and mitochondrial proteins during training in human skeletal muscle. J Physiol. 2010;588(23):4795-810

26. Timmons JA, Szkop KJ, Gallagher IJ. Multiple sources of bias confound functional enrichment analysis of global-omics data. Genome Biol. 2015;16(1):1.

27. McGivney BA, Eivers SS, MacHugh DE, MacLeod JN, O'Gorman GM, Park SD, Katz LM, Hill EW. Transcriptional adaptations following exercise in thoroughbred horse skeletal muscle highlights molecular mechanisms that lead to muscle hypertrophy. BMC Genomics. 2009;10(1):1.

28. McGivney BA, McGettigan PA, Browne JA, Evans AC, Fonseca RG, Loftus BJ, Lohan A, MacHugh DE, Murphy BA, Katz LM. Characterization of the equine skeletal muscle transcriptome identifies novel functional responses to exercise training. BMC Genomics. 2010;11(1):1.
29. Murphy B, Wagner A, McGlynn O, Kharazyan F, Browne J, Elliott J. Exercise influences circadian gene expression in equine skeletal muscle. Vet J. 2014; 201(1):39-45.

30. Eivers S, McGivney B, Gu J, MacHugh D, Katz L, Hill E. PGC-1 a encoded by the PPARGC1A gene regulates oxidative energy metabolism in equine skeletal muscle during exercise. Anim Genet. 2012;43(2):153-62.

31. Hill E, Eivers S, McGivney B, Fonseca R, Gu J, Smith N, Browne J, MacHugh D, Katz L. Moderate and high intensity sprint exercise induce differential responses in COX412 and PDK4 gene expression in thoroughbred horse skeletal muscle. Equine Vet J. 2010;42(s38):576-81.

32. Eivers SS, McGivney BA, Fonseca RG, MacHugh DE, Menson K, Park SD, Rivero J-LL, Taylor CT, Katz LM, Hill EW. Alterations in oxidative gene expression in equine skeletal muscle following exercise and training. Physiol Genomics. 2010;40(2):83-93.

33. Breuer K, Foroushani AK, Laird MR, Chen C, Sribnaia A, Lo R, Winsor GL, Hancock RE, Brinkman FS, Lynn DJ. InnateDB: systems biology of innate immunity and beyond - recent updates and continuing curation. Nucleic Acids Res. 2012;41(D1):D1228-D1233.

34. Charitou T, Bryan K, Lynn DJ. Using biological networks to integrate, visualize and analyze genomics data. Genet Sel Evol. 2016;48(1):1.

35. Mitra K, Carvunis A-R, Ramesh SK, Ideker T. Integrative approaches for finding modular structure in biological networks. Nat Rev Genet. 2013;14(10):719-32.

36. Hartwell LH, Hopfield JJ, Leibler S, Murray AW. From molecular to modular cell biology. Nature. 1999;402:C47-52.

37. Kwon Y, Vinayagam A, Sun X, Dephoure N, Gygi SP, Hong P, Perrimon N. The hippo signaling pathway interactome. Science. 2013;342(6159):737-40.

38. Ito T, Chiba T, Ozawa R, Yoshida M, Hattori M, Sakaki Y. A comprehensive two-hybrid analysis to explore the yeast protein interactome. Proc Natl Acad Sci. 2001;98(8):4569-74

39. Bansal M, Belcastro V, Ambesi-Impiombato A, Di Bernardo D. How to infer gene networks from expression profiles. Mol Syst Biol. 2007;3(1):78.

40. Segal E, Shapira M, Regev A, Pe'er D, Botstein D, Koller D, Friedman N. Module networks: identifying regulatory modules and their conditionspecific regulators from gene expression data. Nat Genet. 2003;34(2):166-76.

41. Segal $E$, Wang $H$, Koller D. Discovering molecular pathways from protein interaction and gene expression data. Bioinformatics. 2003; 19(suppl 1):i264-72.

42. Barabasi A-L, Oltvai ZN. Network biology: understanding the cell's functional organization. Nat Rev Genet. 2004;5(2):101-13.

43. Padi M, Quackenbush J. Integrating transcriptional and protein interaction networks to prioritize condition-specific master regulators. BMC Syst Biol. 2015;9(1):1.

44. Jeong H, Mason SP, Barabási A-L, Oltvai ZN. Lethality and centrality in protein networks. Nature. 2001;411(6833):41-2.

45. Clauset A, Newman ME, Moore C. Finding community structure in very large networks. Phys Rev E. 2004;70(6):066111.

46. Pons $\mathrm{P}$, Latapy M. Computing communities in large networks using random walks. In: International Symposium on Computer and Information Sciences. Istanbul, Turkey - October 26-28, 2005. p. 284-293.

47. Goenawan $1 \mathrm{H}$, Bryan K, Lynn DJ. DyNet: visualization and analysis of dynamic molecular interaction networks. Bioinformatics. 2016;32(17):2713-5.

48. Kerrien S, Aranda B, Breuza L, Bridge A, Broackes-Carter F, Chen C, Duesbury M, Dumousseau M, Feuermann M, Hinz U. The IntAct molecular interaction database in 2012. Nucleic Acids Res. 2011;40(Database issue):D841-6.

49. Xia J, Gill EE, Hancock RE. NetworkAnalyst for statistical, visual and networkbased meta-analysis of gene expression data. Nat Protoc. 2015;10(6):823-44.

50. Ideker T, Ozier O, Schwikowski B, Siegel AF. Discovering regulatory and signalling circuits in molecular interaction networks. Bioinformatics. 2002; 18(suppl 1):S233-40.

51. Li M, Wu X, Wang J, Pan Y. Towards the identification of protein complexes and functional modules by integrating PPI network and gene expression data. BMC Bioinformatics. 2012;13(1):1.

52. Eisen MB, Spellman PT, Brown PO, Botstein D. Cluster analysis and display of genome-wide expression patterns. Proc Natl Acad Sci. 1998;95(25):14863-8.

53. Valette J, Barrey E, JOUGLIN M, COUROUCE A, Auvinet B, Flaux B. Standardisation of muscular biopsy of gluteus medius in French trotters. Equine Vet J. 1999;31(S30):342-4.

54. Krueger F, Kreck B, Franke A, Andrews SR. FastQC: a quality control tool for high throughput sequence data. Nature methods. 2012;9(2):145-51.

55. Dobin A, Davis CA, Schlesinger F, Drenkow J, Zaleski C, Jha S, Batut $P$, Chaisson M, Gingeras TR. STAR: ultrafast universal RNA-seq aligner. Bioinformatics. 2013;29(1):15-21. 
56. Li H, Handsaker B, Wysoker A, Fennell T, Ruan J, Homer N, Marth G, Abecasis $\mathrm{G}$, Durbin R. The sequence alignment/map format and SAMtools. Bioinformatics. 2009;25(16):2078-9.

57. Liao Y, Smyth GK, Shi W. FeatureCounts: an efficient general purpose program for assigning sequence reads to genomic features. Bioinformatics. 2014;30(7):923-30.

58. Ritchie ME, Phipson B, Wu D, Hu Y, Law CW, Shi W, Smyth GK. limma powers differential expression analyses for RNA-sequencing and microarray studies. Nucleic Acids Res. 2015;43(7):e47.

59. Robinson MD, McCarthy DJ, Smyth GK. EdgeR: a Bioconductor package for differential expression analysis of digital gene expression data. Bioinformatics. 2010;26(1):139-40.

60. Kasprzyk A. BioMart: driving a paradigm change in biological data management. Database. 2011;2011:bar049.

61. Ashburner M, Ball CA, Blake JA, Botstein D, Butler H, Cherry JM, Davis AP, Dolinski K, Dwight SS, Eppig JT. Gene ontology: tool for the unification of biology. Nat Genet. 2000;25(1):25-9.

62. Croft D, Mundo AF, Haw R, Milacic M, Weiser J, Wu G, Caudy M, Garapati P, Gillespie M, Kamdar MR. The Reactome pathway knowledgebase. Nucleic Acids Res. 2014;42(D1):D472-7.

63. Kanehisa M, Araki M, Goto S, Hattori M, Hirakawa M, Itoh M, Katayama T, Kawashima S, Okuda S, Tokimatsu T. KEGG for linking genomes to life and the environment. Nucleic Acids Res. 2008;36(suppl 1):D480-4.

64. Fresno C, Fernández EA. RDAVIDWebService: a versatile $R$ interface to DAVID. Bioinformatics. 2013;29(21):2810-1.

65. Hosack DA, Dennis G, Sherman BT, Lane HC, Lempicki RA. Identifying biological themes within lists of genes with EASE. Genome Biol. 2003;4(10):1.

66. Orchard S, Kerrien S, Abbani S, Aranda B, Bhate J, Bidwell S, Bridge A, Briganti L, Brinkman FS, Cesareni G. Protein interaction data curation: the international molecular exchange (IMEx) consortium. Nat Meth. 2012;9(4): 345-50.

67. del-Toro N, Dumousseau M, Orchard S, Jimenez RC, Galeota E, Launay G, Goll J, Breuer K, Ono K, Salwinski L. A new reference implementation of the PSICQUIC web service. Nucleic Acids Res. 2013;41(W1):W601-6.

68. Csardi G, Nepusz T. The igraph software package for complex network research. InterJ, Complex Systems. 2006;1695(5):1-9.

69. Opsahl T, Agneessens F, Skvoretz J. Node centrality in weighted networks: generalizing degree and shortest paths. Soc Networks. 2010;32(3):245-51.

70. Oliver T, Berg JS, Cheney RE. Tails of unconventional myosins. Cell Mol Life Sci. 1999:56(3-4):243-57.

71. Fortier L, Kornatowski M, Mohammed H, Jordan M, O'cain L, Stevens W. Age-related changes in serum insulin-like growth factor-l, insulin-like growth factor-l binding protein-3 and articular cartilage structure in thoroughbred horses. Equine Vet J. 2005;37(1):37-42.

72. Barres R, Yan J, Egan B, Treebak JT, Rasmussen M, Fritz T, Caidahl K, Krook A, O'Gorman DJ, Zierath JR. Acute exercise remodels promoter methylation in human skeletal muscle. Cell Metab. 2012;15(3):405-11.

73. Kitaoka Y, Mukai K, Aida H, Hiraga A, Masuda H, Takemasa T, Hatta H. Effects of high-intensity training on lipid metabolism in thoroughbreds. Am J Vet Res. 2012;73(11):1813-8.

74. Rodgers JT, Lerin C, Haas W, Gygi SP, Spiegelman BM, Puigserver P. Nutrient control of glucose homeostasis through a complex of PGC-1a and SIRT1. Nature. 2005;434(7029):113-8.

75. Lin J, Wu H, Tarr PT, Zhang C-Y, Wu Z, Boss O, Michael LF, Puigserver P, Isotani $\mathrm{E}$, Olson EN. Transcriptional co-activator PGC-1a drives the formation of slow-twitch muscle fibres. Nature. 2002;418(6899):797-801.

76. Lucia A, Gómez-Gallego F, Barroso I, Rabadán M, Bandrés F, San Juan AF, Chicharro JL, Ekelund U, Brage S, Earnest CP. PPARGC1A genotype (Gly482Ser) predicts exceptional endurance capacity in European men. J Appl Physiol. 2005;99(1):344-8.

77. Ling C, Del Guerra S, Lupi R, Rönn T, Granhall C, Luthman H, Masiello P, Marchetti P, Groop L, Del Prato S. Epigenetic regulation of PPARGC1A in human type 2 diabetic islets and effect on insulin secretion. Diabetologia. 2008;51(4):615-22.

78. McGivney B, Herdan C, Gough K, Katz L, Hill E. Effect of training on PPARGC1A and FNDC5 gene expression in thoroughbred horses. Equine Vet J 2014, 46(S46):35-35

79. Leek BT, Mudaliar SR, Henry R, Mathieu-Costello O, Richardson RS. Effect of acute exercise on citrate synthase activity in untrained and trained human skeletal muscle. Am J Phys Regul Integr Comp Phys. 2001;280(2):R441-7.
80. Kitaoka Y, Masuda H, Mukai K, Hiraga A, Takemasa T, Hatta H. Effect of training and detraining on monocarboxylate transporter (MCT) 1 and MCT4 in thoroughbred horses. Exp Physiol. 2011;96(3):348-55.

81. McGowan CM, Golland LC, Evans DL, Hodgson DR, Rose RJ. Effects of prolonged training, overtraining and detraining on skeletal muscle metabolites and enzymes. Equine Vet J. 2002;34(S34):257-63.

82. Vigelsø Hansen A, Andersen NB, Dela F. The relationship between skeletal muscle mitochondrial citrate synthase activity and whole body oxygen uptake adaptations in response to exercise training. Int J Physiol, Pathophysiol Pharmacol. 2014;6(2):84-101.

83. Fulco M, Schiltz RL, lezzi S, King MT, Zhao P, Kashiwaya Y, Hoffman E, Veech RL, Sartorelli $V$. Sir2 regulates skeletal muscle differentiation as a potential sensor of the redox state. Mol Cell. 2003:12(1):51-62.

84. Cantó C, Gerhart-Hines Z, Feige JN, Lagouge M, Noriega L, Milne JC, Elliott PJ, Puigserver P, Auwerx J. AMPK regulates energy expenditure by modulating NAD+ metabolism and SIRT1 activity. Nature. 2009; 458(7241):1056-60.

85. White AT, Schenk S. NAD+/NADH and skeletal muscle mitochondrial adaptations to exercise. Am J Physiol-Endocrinol Metab. 2012;303(3):E308-21.

86. Tapscott SJ. The circuitry of a master switch: Myod and the regulation of skeletal muscle gene transcription. Development. 2005;132(12):2685-95.

87. Lippi G, Maffulli N. Biological influence of physical exercise on hemostasis. In: In: Semin Thromb Hemost: 2009: @ Thieme Medical Publishers. New York. p. 269-76

88. Rosenbaum DM, Rasmussen SG, Kobilka BK. The structure and function of G-protein-coupled receptors. Nature. 2009;459(7245):356-63.

89. Colberg SR, Sigal RJ, Yardley JE, Riddell MC, Dunstan DW, Dempsey PC, Horton ES, Castorino K, Tate DF. Physical activity/exercise and diabetes: a position statement of the American Diabetes Association. Diabetes Care. 2016:39(11):2065-79.

90. Correia SC, Santos RX, Perry G, Zhu X, Moreira PI, Smith MA. Mitochondrial importance in Alzheimer's, Huntington's and Parkinson's diseases. In: Neurodegenerative Diseases. Springer US. 2012; p. 205-21.

91. Buchman AS, Bennett DA. Loss of motor function in preclinical Alzheimer's disease. Expert Rev Neurother. 2011;11(5):665-76.

92. Paillard T, Rolland $Y$, de Souto BP. Protective effects of physical exercise in Alzheimer's disease and Parkinson's disease: a narrative review. J Clin Neurol. 2015;11(3):212-9.

93. Chandra A, Sharma A, Calingasan NY, White JM, Shurubor Y, Yang XW, Beal MF, Johri A. Enhanced mitochondrial biogenesis ameliorates disease phenotype in a full-length mouse model of Huntington's disease. Hum Mol Genet. 2016;25(11):2269-82.

94. Huberts $\mathrm{DH}$, van der Klei IJ. Moonlighting proteins: an intriguing mode of multitasking. Biochimica et Biophysica Acta (BBA)-Molecular. Cell Res. 2010; 1803(4):520-5.

95. Reactome Opioid Receptor Pathway: http://www.reactome.org/content/ detail/R-HSA-167427.

96. Leslie JD, Mayor R. Complement in animal development: unexpected roles of a highly conserved pathway. Semin Immunol. Elsevier US 2013; p. 39-46.

97. Pankov R, Yamada KM. Fibronectin at a glance. J Cell Sci. 2002;115(20):3861-3.

98. Crameri RM, Langberg $H$, Magnusson $P$, Jensen $C H$, Schrøder HD, Olesen $J$, Suetta C, Teisner B, Kjaer M. Changes in satellite cells in human skeletal muscle after a single bout of high intensity exercise. J Physiol. 2004;558(1):333-40.

99. Chervin-Pétinot A, Courçon M, Almagro S, Nicolas A, Grichine A, Grunwald D, Prandini $\mathrm{M}-\mathrm{H}$, Huber $\mathrm{P}$, Gulino-Debrac D. Epithelial protein lost in neoplasm (EPLIN) interacts with a-catenin and actin filaments in endothelial cells and stabilizes vascular capillary network in vitro. J Biol Chem. 2012;287(10):7556-72.

100. Carnemolla A, Labbadia JP, Lazell H, Neueder A, Moussaoui S, Bates GP. Contesting the dogma of an age-related heat shock response impairment: implications for cardiac-specific age-related disorders. Hum Mol Genet. 2014;23(14):3641-56.

101. Ahuja N, Schwer B, Carobbio S, Waltregny D, North BJ, Castronovo V, Maechler $\mathrm{P}$, Verdin E. Regulation of insulin secretion by SIRT4, a mitochondrial ADP-ribosyltransferase. J Biol Chem. 2007;282(46):33583-92.

102. Yang $H_{\text {, }}$ Yang $T$, Baur JA, Perez E, Matsui T, Carmona JJ, Lamming DW Souza-Pinto NC, Bohr VA, Rosenzweig A. Nutrient-sensitive mitochondrial NAD+ levels dictate cell survival. Cell. 2007;130(6):1095-107.

103. Clémençon $B$, Babot $M$, Trézéguet $V$. The mitochondrial ADP/ATP carrier (SLC25 family): pathological implications of its dysfunction. Mol Asp Med. 2013;34(2):485-93. 
104. Predmore BL, Lefer DJ, Gojon G. Hydrogen sulfide in biochemistry and medicine. Antioxid Redox Signal. 2012;17(1):119-40.

105. Módis K, Coletta C, Erdélyi K, Papapetropoulos A, Szabo C. Intramitochondrial hydrogen sulfide production by 3-mercaptopyruvate sulfurtransferase maintains mitochondrial electron flow and supports cellular bioenergetics. FASEB J. 2013;27(2):601-11.

106. Carlin RK, Bartelt DC, Siekevitz P. Identification of fodrin as a major calmodulin-binding protein in postsynaptic density preparations. J Cell Biol. 1983;96(2):443-8.

107. Fedorov VB, Goropashnaya AV, Stewart NC, Tøien $\varnothing$, Chang C, Wang H, Yan J, Showe LC, Showe MK, Barnes BM. Comparative functional genomics of adaptation to muscular disuse in hibernating mammals. Mol Ecol. 2014; 23(22):5524-37.

108. Xu R, Andres-Mateos E, Mejias R, MacDonald EM, Leinwand LA, Merriman DK, Fink RH, Cohn RD. Hibernating squirrel muscle activates the endurance exercise pathway despite prolonged immobilization. Exp Neurol. 2013;247:392-401.

109. Brooks NE, Myburgh KH, Storey KB. Myostatin levels in skeletal muscle of hibernating ground squirrels. J Exp Biol. 2011;214(15):2522-7.

110. Buck MJ, Squire TL, Andrews MT. Coordinate expression of the PDK4 gene: a means of regulating fuel selection in a hibernating mammal. Physio Genomics. 2002:8(1):5-13.

111. Titus MA. Motors: unleashing mitochondria. Curr Biol. 2009:19(23):R1076-8.

112. Kremerskothen J, Plaas C, Kindler S, Frotscher M, Barnekow A. Synaptopodin, a molecule involved in the formation of the dendritic spine apparatus, is a dual actin/a-actinin binding protein. J Neurochem. 2005;92(3):597-606.

113. Ly CV, Verstreken P. Mitochondria at the synapse. Neuroscientist. 2006; 12(4):291-9.

114. Brito GC, Andrews DW. Removing bias against membrane proteins in interaction networks. BMC Syst Biol. 2011;5(1):1.

115. Sakakibara I, Santolini M, Ferry A, Hakim V, Maire P. Six homeoproteins and a linc-RNA at the fast MYH locus lock fast myofiber terminal phenotype. PLoS Genet. 2014;10(5):e1004386.

116. Schwaller B, Dick J, Dhoot G, Carroll S, Vrbova G, Nicotera P, Pette D, Wyss A, Bluethmann H, Hunziker W. Prolonged contraction-relaxation cycle of fast-twitch muscles in parvalbumin knockout mice. Am J Phys Cell Phys. 1999;276(2):C395-403.

117. Boyer-Guittaut M, Poillet L, Liang Q, Bôle-Richard E, Ouyang X, Benavides GA, Chakrama F-Z, Fraichard A, Darley-Usmar VM, Despouy G. The role of GABARAPL1/GEC1 in autophagic flux and mitochondrial quality control in MDA-MB-436 breast cancer cells. Autophagy. 2014;10(6):986-1003.

118. Bruchas MR, Chavkin C. Kinase cascades and ligand-directed signaling at the kappa opioid receptor. Psychopharmacology. 2010;210(2):137-47.

119. Land BB, Bruchas MR, Lemos JC, Xu M, Melief EJ, Chavkin C. The dysphoric component of stress is encoded by activation of the dynorphin K-opioid system. J Neurosci. 2008;28(2):407-14.

120. Bruchas M, Land B, Chavkin C. The dynorphin/kappa opioid system as a modulator of stress-induced and pro-addictive behaviors. Brain Res. 2010;1314:44-55.

121. Ventura B, Genova ML, Bovina C, Formiggini G, Lenaz G. Control of oxidative phosphorylation by Complex I in rat liver mitochondria: implications for aging. Biochimica et Biophysica Acta (BBA)-Bioenergetics. 2002;1553(3):249-60.

122. Castets $P$, Frank S, Sinnreich M, Rüegg MA. "Get the Balance Right": Pathological Significance of Autophagy Perturbation in Neuromuscular Disorders. J Neuromuscul Dis. 2016:3(2):127-55.

123. Schwalm C, Jamart C, Benoit N, Naslain D, Prémont C, Prévet J, Van Thienen $R$, Deldicque L, Francaux M. Activation of autophagy in human skeletal muscle is dependent on exercise intensity and AMPK activation. FASEB J. 2015;29(8): 3515-26.

124. Masiero E, Agatea L, Mammucari C, Blaauw B, Loro E, Komatsu M, Metzger D, Reggiani C, Schiaffino S, Sandri M. Autophagy is required to maintain muscle mass. Cell Metab. 2009;10(6):507-15.

125. Lee J, Giordano S, Zhang J. Autophagy, mitochondria and oxidative stress: cross-talk and redox signalling. Biochem J. 2012;441(2):523-40.

126. He C, Bassik MC, Moresi V, Sun K, Wei Y, Zou Z, An Z, Loh J, Fisher J, Sun Q. Exercise-induced $B C L 2$-regulated autophagy is required for muscle glucose homeostasis. Nature. 2012;481(7382):511-5.

127. Nair $\mathrm{U}$, Klionsky DJ. Activation of autophagy is required for muscle homeostasis during physical exercise. Autophagy. 2011;7(12):1405-6.
128. Delbridge LM, Mellor KM, Taylor DJ, Gottlieb RA. Myocardial autophagic energy stress responses-macroautophagy, mitophagy, and glycophagy. Am J Phys Heart Circ Phys. 2015;308(10):H1194-204.

129. Bruchas MR, Macey TA, Lowe JD, Chavkin C. Kappa opioid receptor activation of p38 MAPK is GRK3-and arrestin-dependent in neurons and astrocytes. J Biol Chem. 2006;281(26):18081-9.

130. Cheng Z-J, Yu Q-M, Wu Y-L, Ma L, Pei G. Selective interference of $\beta$-arrestin 1 with $\mathrm{K}$ and $\delta$ but not $\mu$ opioid receptor/G protein coupling. J Biol Chem. 1998:273(38):24328-33.

131. Desagher S, Glowinski J, Prémont J. Pyruvate protects neurons against hydrogen peroxide-induced toxicity. J Neurosci. 1997;17(23):9060-7.

132. Kaelin WG, Ratcliffe PJ. Oxygen sensing by metazoans: the central role of the HIF hydroxylase pathway. Mol Cell. 2008;30(4):393-402.

133. Kam AY, Chan AS, Wong YH. Phosphatidylinositol-3 kinase is distinctively required for $\mu$-, but not $\mathrm{k}$-opioid receptor-induced activation of $\mathrm{c}$-Jun N-terminal kinase. J Neurochem. 2004;89(2):391-402.

134. Kaneto H, Xu G, Fujii N, Kim S, Bonner-Weir S, Weir GC. Involvement of c-Jun N-terminal kinase in oxidative stress-mediated suppression of insulin gene expression. J Biol Chem. 2002;277(33):30010-8.

135. Delavar H, Nogueira L, Wagner PD, Hogan MC, Metzger D, Breen EC. Skeletal myofiber VEGF is essential for the exercise training response in adult mice. Am J Phys Regul Integr Comp Phys. 2014;306(8):R586-95.

136. Nakatogawa H, Suzuki K, Kamada Y, Ohsumi Y. Dynamics and diversity in autophagy mechanisms: lessons from yeast. Nat Rev Mol Cell Biol. 2009; 10(7):458-67.

137. Zhang Z, Miah M, Culbreth M, Aschner M. Autophagy in neurodegenerative diseases and metal neurotoxicity. Neurochem Res. 2016;41(1-2):409-22.

\section{Submit your next manuscript to BioMed Central and we will help you at every step:}

- We accept pre-submission inquiries

- Our selector tool helps you to find the most relevant journal

- We provide round the clock customer support

- Convenient online submission

- Thorough peer review

- Inclusion in PubMed and all major indexing services

- Maximum visibility for your research

Submit your manuscript at www.biomedcentral.com/submit 